\title{
Molecular-dynamics study of the dynamical excitations in commensurate monolayer films of nitrogen molecules on graphite: A test of the corrugation in the nitrogen- graphite potential
}

\author{
Hansen, Flemming Yssing; Bruch, Ludwig Walter
}

Published in:

Physical Review B

Link to article, DOI:

10.1103/PhysRevB.51.2515

Publication date:

1995

Document Version

Publisher's PDF, also known as Version of record

Link back to DTU Orbit

Citation (APA):

Hansen, F. Y., \& Bruch, L. W. (1995). Molecular-dynamics study of the dynamical excitations in commensurate monolayer films of nitrogen molecules on graphite: A test of the corrugation in the nitrogen-graphite potential. Physical Review B, 51(4), 2515-2536. https://doi.org/10.1103/PhysRevB.51.2515

\section{General rights}

Copyright and moral rights for the publications made accessible in the public portal are retained by the authors and/or other copyright owners and it is a condition of accessing publications that users recognise and abide by the legal requirements associated with these rights.

- Users may download and print one copy of any publication from the public portal for the purpose of private study or research.

- You may not further distribute the material or use it for any profit-making activity or commercial gain

- You may freely distribute the URL identifying the publication in the public portal 


\title{
Molecular-dynamics study of the dynamical excitations in commensurate monolayer films of nitrogen molecules on graphite: A test of the corrugation in the nitrogen-graphite potential
}

\author{
F.Y. Hansen \\ Department of Physical Chemistry, Technical University of Denmark, DTU 206, DK-2800, Lyngby, Denmark \\ L.W. Bruch \\ Department of Physics, University of Wisconsin-Madison, Madison, Wisconsin 53706
}

(Received 19 September 1994)

\begin{abstract}
The dynamical excitations in a commensurate monolayer solid of $\mathrm{N}_{2}$ molecules adsorbed on graphite have been studied using molecular-dynamics simulations. Velocity and rotational correlation functions as well as coherent intermediate scattering functions and dynamical structure factors have been calculated at several temperatures and wave vectors. Effects of out-of-plane motions and the formation of pinwheel-like structural elements associated with the orientational-disorder transition are observed both in the equilibrium order parameters and in the time-correlation functions. The calculated temperature dependence of the Brillouin-zone-center frequency gap in the acoustic phonon branches up to the melting point agrees well with the experimental observations and gives strong support to a model of the substrate corrugation energy. Diffusive motion in the fluid just above the melting point has been demonstrated from the time dependence of the mean-square displacement of the molecules and the two-dimensional diffusion coefficient is estimated to be 2-3 $\times 10^{-5} \mathrm{~cm}^{2} / \mathrm{s}$ at $75-80 \mathrm{~K}$.
\end{abstract}

\section{INTRODUCTION}

Over the past 15-20 years static and dynamical phenomena in physisorbed films of molecules and atoms have been studied with a broad range of experimental and theoretical techniques. ${ }^{1}$ Molecular nitrogen adsorbed on the basal plane surface of graphite is the most extensively studied of the adsorbed molecular solids. There has been a steady development in the ability to model complex phases such as plastic molecular solids; quantitative studies now require establishing the accuracy of the interaction models.

The interactions naturally fall into two categories, namely, interactions between the adsorbed species and between species and substrate. The former, with its orientational dependences, is usually the better known because the major terms are known from three-dimensional (3D) systems. In contrast, the molecule-substrate potential is specific to the system studied and it is difficult to establish systematic trends. For physisorbed systems, the molecule-substrate interaction conventionally is divided into two parts, a large laterally averaged holding potential and a small laterally periodic component usually referred to as the corrugation of the holding potential. For $\mathrm{N}_{2}$ on graphite, the former is $\sim 1100 \mathrm{~K} /$ molecule, whereas the magnitude of the latter is $\sim 20 \mathrm{~K} / \mathrm{molecule}$. The lateral average of the holding potential is determined from experimental energies of adsorption, but it has been very difficult to isolate the corrugation energy experimentally. Since properties of physisorbed films reflect a delicate balance between intermolecular interactions and the lateral corrugation energy, it is important for the understanding of the structure and phase transitions in these films that the lateral variations in the holding potential are known accurately.

Most of our knowledge about the corrugation is based on empirical models; it has only been possible to measure the corrugation experimentally in a few cases. The occurrence of commensurate lattices, such as for krypton on graphite, has been one test of the knowledge. ${ }^{2,3}$ Inelastic neutron scattering data have, however, provided a direct measure of the corrugation in the holding potential for commensurate films of some simple molecules and atoms. The acoustic branches of a crystalline monolayer on a flat surface have zero frequency modes at wave vectors corresponding to the centers of Brillouin zones of the lattice. Such modes correspond to a uniform translation of the entire monolayer. Corrugation of the holding potential gives rise to frustrated or hindered translational motions. In the special case of a monolayer that is commensurate with the substrate, the corrugation provides a coherent restoring force for displacements parallel to the surface and a gap develops in the acoustic branches of the adlayer frequency spectrum. The nonzero frequency at reciprocal lattice vectors of the monolayer solid is usually referred to as the Brillouin-zone-center frequency gap. In spite of the fact that the energy quanta of this gap frequency are rather small, recent inelastic neutron scattering experiments have determined the gap as a function of temperature from very low temperatures (a few degrees Kelvin) up to the melting point for commensurate monolayer solids of $\mathrm{H}_{2}, \mathrm{D}_{2}, \mathrm{HD},{ }^{3} \mathrm{He}, \mathrm{N}_{2}$, and $\mathrm{CD}_{4}$ on graphite. ${ }^{4}$ Results of model calculations of the gap fre- 
quency have an encouraging level of agreement with the low temperature data, except for the $\mathrm{N}_{2}$ system, where initially there was a discrepancy of nearly $50 \% .^{5}$

We have studied the structure, dynamics, and thermal evolution of the commensurate monolayer solid of $\mathrm{N}_{2}$ /graphite. The most uncertain component of the interaction model has been the corrugation energy and we have used the inelastic neutron scattering data to test corrugation models. While it has long been recognized that the geometry of the monolayer imposes a frustration on the ordering of molecular degrees of freedom relative to that in the $3 \mathrm{D}$ solid, we discovered ${ }^{6}$ that for some models of the corrugation there is another significant effect of frustration: The zone-center gap may be small because the molecule-molecule interactions cause the monolayer solid to be positioned on the surface in such a way that the individual molecules are not at minima of the holding potential. In previous work we developed and tested a corrugation model using neutron data for the low-temperature monolayer solid. It was left to follow the thermal evolution of the system to test the model. Results of the test are presented here: the conditions range from the harmonic orientationally ordered solid through a plastic crystal phase to the solid at melting.

In our first study of the inelastic neutron scattering results $^{5}$ we used lattice dynamics calculations to show that existing models, which reproduced many properties of the monolayer solid, gave gap frequencies which were only about half of the observed value. Attempts to force a fit to the observed gap, which worked within a family of models used for adsorbed inert gases, were unsuccessful. Then we implemented ${ }^{6}$ a suggestion of Vernov and Steele $^{7}$ that electrostatic terms make a large contribution to the substrate corrugation for molecules with permanent moments. However, we found an unanticipated competition between electrostatic and van der Waals contributions to the corrugation for the graphite quadrupole terms proposed by Vernov and Steele resulting in a lowering of the gap frequency. When we used the charge density determined for bulk graphite by $\mathrm{x}$-ray diffraction ${ }^{8}$ and represented the electrostatic field as arising from effective quadrupole moments of $\sim 1.0 \times 10^{-26}$ esu $\mathrm{cm}^{2}$ at the position of the $\mathrm{C}$ atoms, the calculated gap frequency was only $\sim 25 \%$ below the experimental value. ${ }^{6}$ This quadrupole moment was of the same order of magnitude as that proposed by Vernov and Steele, but of opposite sign. Then instead of an offsetting effect of the van der Waals and electrostatic contributions to the corrugation, the two mechanisms acted together to enhance the corrugation. Although we could have forced agreement with the experiments by adjusting the surface quadrupole to $\sim 1.5 \times 10^{-26} \mathrm{esu} \mathrm{\textrm {cm } ^ { 2 }}$, we refrained from doing so because that would obscure the point that this interaction was determined independently of the nitrogen system being studied.

Here we complete the tests of the corrugation in the holding potential of the $\mathrm{N}_{2}$ /graphite system by treating the commensurate monolayer solid at temperatures up to melting. This is the most thorough treatment of the dynamics of this system of which we are aware and is based on extensive molecular-dynamics (MD) simulations. We have previously performed a calculation of the static structure of the film and a lattice dynamics calculation of the intermediate scattering function and the signature of the frequency gap in inelastic neutron scattering. Now we use a MD calculation to determine the frequency gap as a function of temperature up to the melting point. There is good agreement with inelastic neutron scattering results all the way up to the melting point. We use the detailed picture contained in the time-correlation functions to confirm the evolution of correlations and structure that has been inferred from equilibrium properties.

Our work supplements and amplifies observations made in other work on the dynamics of adsorbed molecular solids. There is a considerable overlap with the Monte Carlo simulations of Etters and his co-workers. ${ }^{9,10}$ They use a more complex model of the molecule-molecule interaction, but there is good agreement of results from the two approaches. There remains uncertainty about the contributions of substrate-mediated interactions. While such terms are clearly present for $\mathrm{N}_{2} /$ graphite, ${ }^{11}$ the model used may be incomplete. The pioneering MD calculation for this system was done by Cardini and $\mathrm{O}^{\prime}$ Shea ${ }^{12}$ before the need for drastic modification to the corrugation model was known. Some of the dynamical features associated with the orientational disorder transition were first noted in MD simulations of Lynden-Bell et $a l .{ }^{13}$ Another observation is that the azimuthal angle disorder transition is coupled to the occurrence of nonplanar orientations, pinwheel-like configurations, in the thermally excited films; related observations have been made in computer simulations of denser nitrogen monolayer solids, ${ }^{14}$ but they were not linked to the disorder transition. The excitation of out-of-plane orientations softens the structure and makes room for the other molecules to disorder in the azimuthal angle. Our analysis of the calculations for the intermediate scattering function and the response to inelastic neutron scattering has many parallels in the work of Shrimpton and Steele ${ }^{15}$ for a monatomic lattice.

The organization of the paper is as follows. Section II describes the methods used in the calculations. It includes a solution to the problem of determining effective two-center forces, one at each nitrogen atom position, from the set of forces arising in the distributed point charge model of the molecular quadrupole moment. It also contains a description of the calculation of velocity and rotational time-correlation functions and of the coherent intermediate scattering functions. Section III contains a summary of the interaction model. Section IV presents results for the equilibrium properties, with an emphasis on the qualitative changes in molecular motions at the orientational disorder transition and at the melting transition. Section V presents results for the various velocity and rotational correlation functions, for the intermediate scattering functions, and for zone-center frequency gap as a function of the temperature. Section VI has some concluding remarks and a discussion of systematic trends for the nitrogen and adsorbed hydrocarbon series. 


\section{CALCULATIONAL METHODS}

We performed isothermal molecular-dynamics simulations with a constrained molecular degree of freedom, using Gear's fourth-order predictor corrector algorithm to integrate the equations of motion. ${ }^{16}$ The diatomic $\mathrm{N}_{2}$ molecule is described by the six Cartesian coordinates of the atoms and the internuclear distance is held fixed by using a constraint dynamics algorithm in the equations of motion. ${ }^{17}$ The time step in the integration was determined from a microcanonical (constant energy) simulation; a time step of 0.0025 ps gave a satisfactory energy conservation with only a small drift of $\sim 0.009 \% / \mathrm{ps}$.

The initial configuration in all simulations was the commensurate rectangular herringbone structure with lattice vectors $7.38 \AA$ and $4.26 \AA$ which is the low temperature state of the molecular solid. The unit cell in the simulation is an almost square simulation box with eight 7.38- $\AA$ lattice vectors along the $x$ axis $(59.040 \AA)$ and fourteen 4.26 - $\AA$ lattice vectors along the $y$ axis $(59.652$ $\AA)$; it contains 224 molecules. These lengths are on the scale of the coherence length $\sim 90-150 \AA$ inferred for the graphite substrate in neutron scattering experiments on monolayer films of butane and hexane. ${ }^{18}$ Periodic boundary conditions were imposed only along the $x$ and $y$ axes, parallel to the surface. The graphite substrate is treated as a static lattice; it provides the external (holding) potential for the molecules and participates as a third body in molecule-molecule dispersion energies.

When a molecule passed the point of no return along $z$, defined as the distance $\left(=3.5 \sigma_{\mathrm{NC}}\right)$, where the holding potential is $1 \%$ of the well depth in the nitrogen-carbon potential [Eq. (20)], its velocity in the $z$ direction was inverted to bring it back to the surface. Desorption of molecules became noticeable at temperatures of about 75 $\mathrm{K}$ in the present work and times of the order of several hundred picoseconds were required for equilibration.

For the determination of structural properties and thermodynamic functions, the system was propagated in time blocks of $50 \mathrm{ps}$ for a total time of at least $200 \mathrm{ps}$. At the completion of each time block averages were calculated for that time block as well as accumulated averages over the different time blocks. This enabled an estimate of the statistical uncertainty in the averages. By monitoring the series of structural and thermodynamic data in each time block it also was fairly easy to assess when the system had equilibrated.

The system was typically propagated for $200 \mathrm{ps}$, but near a phase transition the total time was at least 600 ps. There are signatures in the calculations for two phase transitions: orientational disordering of the molecules and melting of the commensurate lattice. Here the transitions are manifested by instability of single-phase simulations and may be subject to metastability problems. However, other workers using single-phase treatments of melting for closely related monolayer models have found (i) agreement with the melting condition determined by free-energy constructions ${ }^{19}$ and (ii) no hysteresis in carefully equilibrated simulations. ${ }^{9}$ It is known ${ }^{20}$ that more detailed constructions are needed to avoid metastability effects in the melting of three-dimensional bulk $\mathrm{N}_{2}$.
The monolayer orientational-disorder transition is either a continuous transition or a weak first-order transition; to decide that in a simulation requires much larger samples than those used here. ${ }^{21}$

The data for calculation of time-correlation functions were generated by continuing the calculations for a further time period after the equilibrium properties were determined, typically after 200 ps. The time-correlation functions are generally of the form

$$
C\left(t-t_{0}\right)=\left\langle F(t) F\left(t_{0}\right)\right\rangle /\left\langle F\left(t_{0}\right) F\left(t_{0}\right)\right\rangle,
$$

where $F(t)$ is some molecular or atomic property at time $t$ and $C(\tau)$ the time-correlation function at time $\tau$ normalized to one at time $\tau=0$. A sequence of positions and velocities is collected at some specified time interval, where the interval and time period are dictated by the frequency spectrum. The length of the period depends on the desired frequency resolution in Fourier spectra and the time interval between the storage of position and velocity data depends on the time scales in the correlation function considered.

The correlation functions in Eq. (1) are calculated following the method described by Allen and Tildesley. ${ }^{22}$ The initial time $t_{0}$ is any of the times in the sequence of the stored data and the correlation with later times is formed as an average over all molecules and over initial times. To reduce the statistical correlation of successive averages, an interval is maintained between choices of initial times in the stored data. To maintain comparable statistical accuracy at all times $\tau$ we usually calculated the time-correlation functions to a shorter time than the total time interval $T_{\max }$ of the stored data.

For the calculation of the correlation functions and the intermediate scattering function, the system was propagated for $40 \mathrm{ps}$ with a time interval $\delta t=0.125 \mathrm{ps}$ between storage of position and velocity data. The time correlation functions were calculated up to $20 \mathrm{ps}$, i.e., a frequency resolution of $0.05 \mathrm{THz}$ compared to a minimum frequency in the spectrum of the intermediate scattering function, which is in the range of $0.25-0.4 \mathrm{THz}$. The time interval of 0.125 ps corresponds to a maximum frequency of $4 \mathrm{THz}$; lattice dynamical calculations for the monolayer model showed the maximum frequency to be about $2 \mathrm{THz} .^{5,6}$

\section{A. Effective forces}

In the molecular dynamics we treat the motions of the two atomic centers under local forces, but the electrostatic forces between the molecules are modeled using distributed point charges. Therefore the electrostatic forces acting on the point charges must be converted to forces acting on the two atomic centers. For a rigid molecule, this is done by requiring that the effective forces at the atomic sites give the same total force and torque on the molecule as the sum of the electrostatic terms.

Assume that there are $m$ point charges at positions $\mathbf{s}_{i}$ relative to the center of mass of the molecule and let the unit vector in the direction from atom 1 to atom 2 be 
$\hat{\mathbf{n}}$. Let the distance between the nitrogen atoms be $l$ and the Coulombic force at center $\mathrm{j}$ be $f_{j}$. Then the sum of equivalent two-center forces $F_{i}$ must be

$$
\mathbf{F}_{1}+\mathbf{F}_{2}=\sum_{j=1}^{m} \mathbf{f}_{j} \equiv \mathbf{F}
$$

For the rigid molecule, we may also impose the constraint

$$
\hat{\mathbf{n}} \cdot\left(\mathbf{F}_{2}-\mathbf{F}_{1}\right)=0 \text {, }
$$

i.e., there is no differential in the force along the internuclear axis. The torque around the molecular center is given by

$$
\frac{l}{2} \hat{\mathbf{n}} \times\left(\mathbf{F}_{2}-\mathbf{F}_{1}\right)=\sum_{j=1}^{m} \mathbf{s}_{j} \times \mathbf{f}_{j} \equiv \tau .
$$

The solution of this last pair of equations is

$$
\mathbf{F}_{1}-\mathbf{F}_{2}=\frac{2}{l} \hat{\mathbf{n}} \times \boldsymbol{\tau}
$$

and combined with Eq. (2) we find

$$
\begin{aligned}
& \mathbf{F}_{1}=\frac{1}{2} \mathbf{F}+\frac{1}{l} \hat{\mathbf{n}} \times \boldsymbol{\tau}, \\
& \mathbf{F}_{2}=\frac{1}{2} \mathbf{F}-\frac{1}{l} \hat{\mathbf{n}} \times \boldsymbol{\tau} .
\end{aligned}
$$

Thus the total force and torque from the Coulombic interactions, $\mathbf{F}$ and $\tau$, are reexpressed as the equivalent two-center forces $F_{1}$ and $F_{2}$ with Eqs. (6) and (7).

\section{B. Time-correlation functions and frequency spectra}

Three types of correlation functions have been considered. The first is the rotational and velocity autocorrelation functions which contain the frequency spectra of the dynamical excitations and the correlation times in the various modes of motion. We evaluated correlation functions for the azimuthal angle $\phi$ and for the polar angle $\theta$ of the N-N bond and for the center of mass velocity correlation functions along the $x, y$, and $z$ axes, separately. The second type is the coherent intermediate scattering function $I_{\operatorname{coh}}(\kappa, t)$ and its time Fourier transform, the coherent scattering function $S_{\mathrm{coh}}(\kappa, \omega)$. In particular, coherent neutron scattering for a wave vector $\kappa$ equal to a reciprocal lattice vector of the monolayer solid leads to a determination of the Brillouin-zone-center frequency gap. The third type is the mean square displacement of the molecular center of mass positions as a function of time. This function is bounded at large times for the solid, but diverges for the fluid.

In the fluid phase the mean square displacement increases linearly with time

$$
\left\langle\left|\mathbf{R}_{i}(t)-\mathbf{R}_{i}\left(t_{0}\right)\right|^{2}\right\rangle \propto t,
$$

where $\mathbf{R}_{i}(t)$ is the position of molecule $i$ at time $t$. For 3D isotropic diffusion in a liquid with diffusion constant $D$, the proportionality constant equals $6 D$, but here the system is highly anisotropic with $2 \mathrm{D}$ diffusion parallel to the surface and mostly bounded vibrational motion in the $z$ direction perpendicular to the surface. Then the proportionality constant is $4 D$, where $D$ is the selfdiffusion constant in the plane.

Below the melting point, correlation function data for 20 ps were sufficient to evaluate the mean square displacement. In the fluid phase much longer times were necessary before the linear time dependence expected for diffusion became apparent and there were complications from the effects of desorbing molecules and redistribution of molecules between layers. For the analysis of diffusion in the fluid, the system was propagated for 1000 ps; molecular configurations for the final 400 ps were retained, with a time interval of 1.25 ps between data storages.

The intermediate scattering function at reciprocal lattice vectors has a dominant elastic contribution, corresponding to Bragg reflections. To detect inelastic contributions from the translational modes associated with the zone-center frequency gap, first the elastic term must be removed. The intermediate scattering function including the elastic term is defined to be $^{23}$

$$
\begin{aligned}
& I_{\operatorname{coh}}(\boldsymbol{\kappa}, t) \\
&=\left\langle\sum_{i} \exp \left[i \kappa \cdot \mathbf{r}_{i}(t+\tau)\right] \sum_{j} \exp \left[-i \kappa \cdot \mathbf{r}_{j}(\tau)\right]\right\rangle
\end{aligned}
$$

where the angular brackets indicate an ensemble average, i.e., an average over initial times $\tau$. The sums are over atomic positions or molecular positions in the simulation cell; we have considered both. To isolate the elastic term, first introduce the function

$$
f(\tau)=\sum_{i} \exp \left[i \kappa \cdot \mathbf{r}_{i}(\tau)\right]
$$

and determine its average $\bar{f}$ over all $N T$ entries in the time sequence of positions and velocities generated in the MD simulation

$$
\bar{f}=\frac{1}{N T} \sum_{\tau=1}^{N T} f(\tau) .
$$

The modified intermediate scattering function is the autocorrelation of $f$ with the time-independent contribution $\bar{f}$ removed

$$
I_{\text {coh }}^{\prime}(\kappa, t)=\left\langle[f(t+\tau)-\bar{f}]\left[f^{*}(\tau)-\bar{f}^{*}\right]\right\rangle,
$$

where the asterisk denotes the complex conjugate function. The modified scattering function differs from the intermediate scattering function by an additive constant, so that the two functions have the same power spectrum except at zero frequency. For the numerical work, elim- 
ination of the constant term reduces spurious spectral density at finite frequencies.

The one-phonon approximation to the scattering function has also been calculated. We begin by reformulating $f(\tau)$ in Eq. (10) in terms of the average position $\overline{\mathbf{r}}_{i}$ of atom $i$ during the time sequence

$$
\begin{gathered}
f(\tau)=\sum_{i} \exp \left\{i \kappa \cdot\left[\mathbf{r}_{i}(t)-\overline{\mathbf{r}}_{i}\right]\right\} \exp \left(i \kappa \cdot \overline{\mathbf{r}}_{i}\right), \\
\overline{\mathbf{r}}_{i}=\frac{1}{N T} \sum_{\tau=1}^{N T} \mathbf{r}_{i}(\tau) .
\end{gathered}
$$

The one-phonon theory arises from a first-order Taylor series expansion in $\mathbf{r}_{i}(t)-\overline{\mathbf{r}}_{i}$. Then, as in Eq. (12), the modified one-phonon scattering function is defined to be

$$
\begin{aligned}
I_{\text {coh }}^{\text {one }}(\kappa, t) \\
=I_{\text {coh }}^{\text {one }}(\kappa, t)-\sum_{i} \exp \left(i \kappa \cdot \overline{\mathbf{r}}_{i}\right) \sum_{j} \exp \left(-i \kappa \cdot \overline{\mathbf{r}}_{j}\right) \\
=\left\langle\sum_{i} \kappa \cdot\left[\mathbf{r}_{i}(t+\tau)-\overline{\mathbf{r}}_{i}\right] \exp \left(i \kappa \cdot \overline{\mathbf{r}}_{i}\right)\right. \\
\left.\quad \times \sum_{j} \kappa \cdot\left[\mathbf{r}_{j}(\tau)-\overline{\mathbf{r}}_{j}\right] \exp \left(-i \kappa \cdot \overline{\mathbf{r}}_{j}\right)\right\rangle .
\end{aligned}
$$

This formulation omits the Debye-Waller factor in the one-phonon response function defined by Maradudin and Fein. ${ }^{24}$

The function $f(\tau)$ in Eq. (10), when evaluated as a sum over all atoms, also enters in the calculation of the structure factor at Bragg reflections, which is defined to be

$$
S\left(\kappa_{i, j}\right) \equiv S(i, j)=\left\langle f(\tau) f^{*}(\tau)\right\rangle
$$

for reciprocal lattice vectors

$$
\kappa_{i, j}=2 \pi[i \hat{\mathbf{x}} / 7.38+j \hat{\mathbf{y}} / 4.26] .
$$

\section{INTERACTION MODEL}

The empirical molecule-molecule interaction inferred from 3D data is supplemented by estimates of adsorptioninduced interactions and by a model of the moleculegraphite interaction. We use the X1 model of Murthy et al. ${ }^{25}$ fitted to $3 \mathrm{D}$ dense phase data. It consists of atom-atom interactions and a distributed point charge model for the electrostatic interactions between nitrogen molecules. The atom-atom potentials are represented by a Lennard-Jones $(12,6)$ potential

$$
E_{\mathrm{NN}}\left(R_{i j}\right)=4 \epsilon_{\mathrm{NN}}\left[\left(\frac{\sigma_{\mathrm{NN}}}{R_{i j}}\right)^{12}-\left(\frac{\sigma_{\mathrm{NN}}}{R_{i j}}\right)^{6}\right],
$$

with $\epsilon_{\mathrm{NN}}=36.4 \mathrm{~K}, \sigma_{\mathrm{NN}}=3.318 \AA$, and distance $R_{i j}$ between atoms $i$ and $j$. The atom-atom interactions are truncated at distances larger than $10 \AA$. The charge dis- tribution in the molecule is represented by three point charges. ${ }^{25}$ The charges are $q=-0.405|e|$ at the atomic centers and $0.810|e|$ at the molecular center, where $e$ is the charge of an electron. The effective molecular quadrupole moment is $-1.173 \times 10^{-26} \mathrm{esu} \mathrm{cm}^{2}$. The distributed point charge model was constructed to include effects of the finite extent of the molecular charge distribution, but the effective quadrupole moment was set to be smaller than that for the isolated molecule, to include effects of penetration of charge clouds in the dense phases.

The presence of the graphite surface, however, modifies the 3D interaction. The substrate is included in our potential model in the form of the substrate mediated dispersion energy given by McLachlan, ${ }^{26}$

$$
\begin{aligned}
E_{i j}^{\mathrm{McL}}= & \frac{C_{S 1}}{\left(R_{i j} R_{i^{\prime} j}\right)^{3}}\left[\frac{4}{3}-\frac{\left(r_{j z}+r_{i z}-z_{\text {lay }}\right)^{2}}{R_{i^{\prime} j}^{2}}\right. \\
& \left.-\frac{\left(r_{j z}-r_{i z}\right)^{2}}{R_{i j}^{2}}\right]-\frac{C_{S 2}}{R_{i^{\prime} j}^{6}},
\end{aligned}
$$

with $C_{S 1}=5.7898 \times 10^{4} \mathrm{~K} \AA^{6}$ and $C_{S 2}=2.9468 \times 10^{4} \mathrm{~K}$ $\AA^{6} .^{27} i^{\prime}$ is the image of atom $i$ in a mirror plane parallel to the surface and shifted $z_{\text {lay }} / 2$ outward, where $z_{\text {lay }}=3.37$ $\AA$ is the distance between the basal planes in graphite. $R_{i j}$ is the distance between atoms $i$ and $j$ in different nitrogen molecules and $r_{i z}$ is the $z$ coordinate of atom $i$. For atoms at equal heights above the surface this term is repulsive and reduces the intermolecular attractive energy by about $15 \%$. For adatoms that are not at equal height, it may enhance the attraction. There is experimental evidence ${ }^{11}$ that the attractive interaction between adsorbed nitrogen molecules is reduced by about $20 \%$, but there is no detailed support for this mechanism being the only significant adsorption-induced interaction. We distribute the McLachlan energy over atom-atom pairs; it must be noted that there is no consistency, even for the same workers, on using the atom-atom distribution or a total acting at the molecular centers.

The molecule-substrate interaction is based on the Lennard-Jones $(12,6)$ atom-atom potential for nitrogengraphite carbon interactions with Steele's parameters $\epsilon_{\mathrm{NC}}=31 \mathrm{~K}$ and $\sigma_{\mathrm{NC}}=3.36 \AA^{28}$ However, we found ${ }^{5}$ that the corrugation of the molecule-substrate potential formed $^{29}$ from such an isotropic atom-atom potential is too small to reproduce the observed zone-center frequency gap. Carlos and Cole ${ }^{30}$ treated a closely related problem for adsorbed inert gases by adding terms that reflect the anisotropy in the polarizability of the graphite substrate. They generalized the atom-atom N-C interaction to be

$$
\begin{aligned}
E_{\mathrm{NC}}\left(R_{i j}\right) & =4 \epsilon_{\mathrm{NC}}\left\{\left(\frac{\sigma_{\mathrm{NC}}}{R_{i j}}\right)^{12}\left[1+\gamma_{R}\left(1-\frac{6}{5} \cos ^{2} \theta\right)\right]\right. \\
& \left.-\left(\frac{\sigma_{\mathrm{NC}}}{R_{i j}}\right)^{6}\left[1+\gamma_{A}\left(1-\frac{3}{2} \cos ^{2} \theta\right)\right]\right\} .
\end{aligned}
$$

Here $\theta$ is the angle between the surface normal and the 
$\mathbf{R}_{i j}$ vector. The modification changes the corrugation of the molecule-substrate potential, but it leaves the lateral average of the potential the same. The two coefficients $\gamma_{A}$ and $\gamma_{R}$ specify the modifications to the van der Waals attraction and the overlap repulsion, respectively. The value $\gamma_{A}=0.4$ is set by the dielectric properties of the graphite while $\gamma_{R}$ is an adjustable parameter. Carlos and Cole $^{30}$ fitted $\gamma_{R}=-0.54$ for helium, and this value may apply for other inert gas atoms. Joshi and Tildesley ${ }^{31}$ fitted $\gamma_{R}=-1.04$ for nitrogen; calculations using this anisotropy $^{5}$ gave a small increase in the zone-center gap frequency, which remained only slightly more than half of the observed value.

The separation of the nitrogen-graphite interaction into a laterally averaged term and corrugation amplitudes is based on Steele's transformation of the atomatom sums. ${ }^{29}$ The translational symmetry of the graphite substrate leads to a Fourier representation for the nitrogen-substrate dispersion interaction as

$$
E_{\text {surf }}^{\text {disp }}(z, \mathbf{r})=E_{0}^{\text {disp }}(z)+\sum_{\mathbf{g}} E_{\mathbf{g}}^{\text {disp }}(z) \exp (i \mathbf{g} \cdot \mathbf{r}),
$$

where $\mathbf{r}$ is the lateral position vector of an $\mathrm{N}$ atom, $z$ is its vertical distance from the graphite surface, and $\mathbf{g}$ are the (2D) reciprocal lattice vectors of the graphite basal planes. Carlos and $\mathrm{Cole}^{30}$ presented expressions for the functions in Eq. (21) for the N-C dispersion potential; we include them here for completeness and for comparison with the electrostatic contributions. The laterally averaged term in the potential, $E_{0}^{\text {disp }}(z)$, is given by

$$
E_{0}^{\mathrm{disp}}\left(z_{\alpha}\right)=\epsilon_{\mathrm{NC}} \frac{2 \pi}{A_{S}} n\left[\frac{2}{5} \frac{\sigma_{\mathrm{NC}}^{12}}{z_{\alpha}^{10}}-\frac{\sigma_{\mathrm{NC}}^{6}}{z_{\alpha}^{4}}\right]
$$

Here $n$ is the number of atoms (equal to 2) in the 2D unit cell of the basal plane. $A_{S}$ is the area of the $2 \mathrm{D}$ unit cell and $z_{\alpha}$ is the vertical distance from the atom to the $\alpha$ th basal plane in the graphite crystal. As noted above, the lateral average of the potential is independent of the $\gamma_{A}$ and $\gamma_{R}$ parameters. The Fourier coefficients $E_{\mathbf{g}}^{\text {disp }}\left(z_{\alpha}\right)$ are given by

$$
\begin{aligned}
E_{\mathbf{g}}^{\mathrm{disp}}\left(z_{\alpha}\right)= & \epsilon_{\mathrm{NC}} \frac{2 \pi}{A_{S}} \sum_{g \neq 0}\left(\left\{\sigma_{\mathrm{NC}}^{12}\left[\frac{1}{30}\left(\frac{g}{2 z_{\alpha}}\right)^{5}\left(1+\gamma_{R}\right) K_{5}\left(g z_{\alpha}\right)-\frac{1}{150} \gamma_{R} z_{\alpha}^{2}\left(\frac{g}{2 z_{\alpha}}\right)^{6} K_{6}\left(g z_{\alpha}\right)\right]\right.\right. \\
& \left.\left.-\sigma_{\mathrm{NC}}^{6}\left[2\left(\frac{g}{2 z_{\alpha}}\right)^{2}\left(1+\gamma_{A}\right) K_{2}\left(g z_{\alpha}\right)+\gamma_{A} z_{\alpha}^{2}\left(\frac{g}{2 z_{\alpha}}\right)^{3} K_{3}\left(g z_{\alpha}\right)\right]\right\} \sum_{n} \exp \left[i \mathrm{~g} \cdot\left(\mathbf{r}-\tau_{n}\right)\right]\right)
\end{aligned}
$$

$K_{i}(x)$ is the $i$ th modified Bessel function and $\tau_{n}$ the 2D position vector of the carbon atoms in the $2 \mathrm{D}$ unit cell of the basal planes of graphite. In practice, 15 layers of basal planes of the graphite crystal are included in the calculation of $E_{0}^{\text {disp }}(z)$ and the corrugation is calculated from the topmost graphite plane and for the first two shells of reciprocal lattice vectors.

In subsequent work $^{6}$ we found a significant increase in the calculated corrugation from a mechanism proposed by Vernov and Steele. ${ }^{7}$ They noted that bonding in the graphite gives rise to aspherical atomic charge distributions which are the source of electrostatic fields with rapid spatial variations external to the graphite. We used x-ray data for the valence charge distribution of bulk graphite $^{8}$ to determine an effective quadrupole moment. The construction of the effective moment from the atomic charge density included terms beyond the quadrupole and is based on data for the bulk charge densities, although there may be some charge relaxation relative to the bulk for the surface atoms which give the dominant contributions to the electric field.

The quadrupole moment tensor for atoms of the graphite is given by

$$
-2 \Theta_{x x}=-2 \Theta_{y y}=\Theta_{z z} \equiv \Theta_{C}
$$

with all other elements equal to zero. The effective quadrupole moment $\Theta_{C}$ in Eq. (24) is defined in terms of the atomic charge density $\rho(s)$ by

$$
\Theta_{C}=\left(2 / g^{2}\right) \int d^{3} \mathbf{s} \rho(\mathbf{s}) \exp \left(g s_{z}-i \mathbf{g} \cdot \mathbf{s}_{\mathrm{par}}\right)
$$

where the vector distance $\mathbf{s}$ from the carbon nucleus is decomposed into components parallel ( $\mathbf{s}_{\text {par }}$ ) and perpendicular $\left(s_{z}\right)$ to the basal plane. The moment $\Theta_{C}$ derived from Eq. (25) is equal to the quadrupole moment $\left(\Theta_{z z}\right)$ for a pure quadrupolar charge distribution aligned along the $z$ axis. For the more general distribution determined in the $x$-ray experiments, the effective moment depends on the reciprocal lattice vector $\mathbf{g}$. Here the leading star of six reciprocal lattice vectors of the graphite (of length $g$ $=2.95 \AA^{-1}$ and with one along the $y$ axis) is used and the octupolar distortion of the graphite carbons, of opposite sense for the two carbons in the graphite unit cell, is included. This leads to the effective value $\Theta_{C}=1.0 \times 10^{-26}$ esu $\mathrm{cm}^{2}$ (Ref. 6) used in our work.

The electrostatic energy $e_{q q}$ between a point charge $q$ and a quadrupole $\Theta$ (Ref. 32) is

$$
e_{q q}=\frac{1}{3} q \sum_{\alpha, \beta} \Theta_{\alpha \beta} \frac{3 R_{\alpha} R_{\beta}-R^{2} \delta_{\alpha \beta}}{R^{5}}
$$

where $\alpha, \beta$ runs over all Cartesian coordinates $x, y, z$ and $R$ is the distance between the charge and the quadrupole. Then the electrostatic energy of a point charge in a nitrogen molecule and a quadrupole at a graphite carbon atom position is 


$$
e_{q q}=\frac{1}{2} q \Theta_{z z}\left[\frac{3 R_{z}^{2}}{R^{5}}-\frac{1}{R^{3}}\right] .
$$

The translation symmetry of the graphite substrate also leads to a Fourier representation of the nitrogensubstrate electrostatic interaction

$$
E_{\text {surf }}^{\mathrm{el}}(z, \mathbf{r})=\sum_{\mathbf{g}} E_{\mathbf{g}}^{\mathrm{el}}(z) \exp (i \mathbf{g} \cdot \mathbf{r})
$$

with the same notation as in Eq. (21). For the two-body interaction energy of Eq. (27), the total interaction energy of a charge in the nitrogen molecule with the substrate quadrupoles is given by

$$
\begin{aligned}
& E_{\text {surf }}^{\mathrm{el}}(z, \mathbf{r}) \\
& \quad=\frac{\pi}{A_{S}} q \Theta_{C} \sum_{n} \sum_{\mathbf{g}} g \exp (-g z) \exp \left[i \mathbf{g} \cdot\left(\mathbf{r}-\tau_{\mathbf{n}}\right)\right] .
\end{aligned}
$$

Vernov and Steele estimated the surface quadrupole moment from data for the aromatic hydrocarbon molecules. They assigned a quadrupole moment at each carbon atom in the graphite surface of $-1.3 \times 10^{-26} \mathrm{esu}$ $\mathrm{cm}^{2}$; it is similar in magnitude but opposite in sign to that derived from the x-ray data. Recently, there has been a report of a measurement for bulk graphite leading to carbon quadrupole moments equal to $-\Theta_{C} \cdot{ }^{33}$ When this last value is used in the calculations at $10 \mathrm{~K}$, it leads to a frustrated layer of a type discussed in our previous work. ${ }^{6}$ The calculated zone-center gap is only about 0.125 $\mathrm{THz}$, much smaller than the observed value of $0.4 \mathrm{THz}$ and even smaller than that with no surface quadrupoles; thus we have not pursued this case further. The discrepancy in sign of the quadrupole moment has not yet been resolved.

The combination of Steele's N-C atom-atom potential, the graphite anisotropy with the Carlos-Cole parameters, and the substrate electrostatic field term brought the calculated gap frequency to within $25 \%$ of the experimental value. ${ }^{6}$ The anisotropy parameters of Joshi and Tildesley ${ }^{31}$ were based on a fit to the melting temperature of submonolayer films of $\mathrm{N}_{2}$. We suggested that their change in $\gamma_{R}$ may have been driven in part by the omission of the substrate electrostatic field; that would have a greater consequence for the quadrupolar molecule than for the isotropic inert gas atoms. Thus the recommended potential model from our previous work had the substrate anisotropy parameters of Carlos and Cole. It remained to calculate the melting conditions for the recommended potential model.

\section{EQUILIBRIUM PROPERTIES}

We have made a series of simulations at temperatures from $10 \mathrm{~K}$ to $80 \mathrm{~K}$ in order to study the equilibrium properties and phase transitions of the solid film. The temperatures span a range from a harmonic solid, well ordered in molecular orientations and centers of mass, through a plastic solid with disordered azimuthal degrees of freedom, and finally to the solid near melting, which has a significant population of thermally excited vacancies.

At low temperatures, the nitrogen molecules form a herringbone structure with two molecules in a rectangular unit cell with dimensions $(7.38 \AA \times 4.26 \AA)$. The molecules are arranged in two sublattices with the $\mathrm{N}-\mathrm{N}$ axes parallel to the surface and the azimuthal angle of the $\mathrm{N}-\mathrm{N}$ axes at approximately $\pm 45^{\circ}$ with respect to the 7.38- $\AA$ side of the rectangular unit cell. At about 20-25 $\mathrm{K}$ there is a transition where the azimuthal orientations disorder but an ordered hexagonal lattice of the molecular centers remains.

The temperature dependence in the region of the transition is shown in Fig. 1, where we have plotted the orientational order parameters $o 1=\left\langle\sin \left(2 \phi_{1}\right)\right\rangle$ and $o 2=$ $\left\langle\sin \left(2 \phi_{2}\right)\right\rangle$ for sublattices 1 and 2 . The angular brackets denote an ensemble average over values of the sine of the azimuthal angles $\phi_{1}$ and $\phi_{2}$ on the two sublattices. The order parameters vary from $o 1=-o 2 \simeq 1$ at low temperatures to zero at high temperatures, where there is nearly free azimuthal rotation. Another order parameter showing the transition is the intensity of the $(1,2)$ Bragg reflection for the herringbone superlattice, which drops drastically in passing through the transition. The position of the inflection point in each of the three order parameter graphs is consistent with a transition temperature of $22 \mathrm{~K}$; this is somewhat lower than the experimental value ${ }^{35}$ of $26-28 \mathrm{~K}$. A possible reason for this is discussed in Sec. VI.

In Fig. 2 the azimuthal angle distribution at several temperatures is shown. The two distinct peaks at low temperature reflect the herringbone sublattices. Just below the transition temperature, there are two extra small peaks at $180^{\circ}$ from the main peaks. Since the ends of the molecules are labeled in the simulation, this shows the beginning of a tendency of molecules to rotate $180^{\circ}$

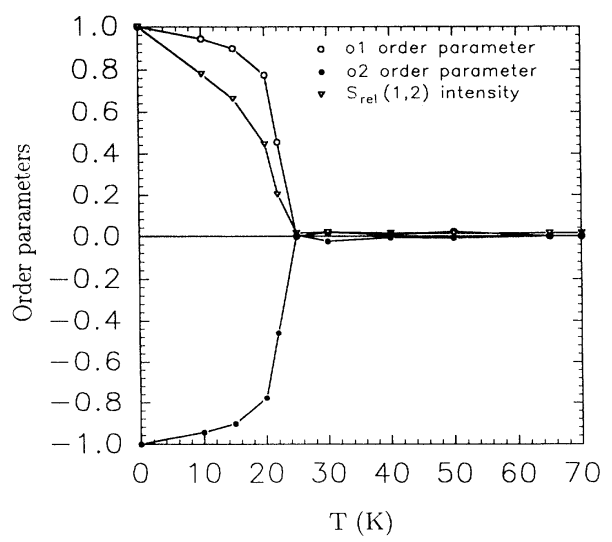

FIG. 1. The temperature dependence of the N-N azimuthal angle order parameters $o 1$ and $o 2$ for sublattices 1 and 2, respectively, and the structure factor $S_{\text {rel }}(1,2)$ of the herringbone lattice, relative to its zero temperature value of $S(1,2)$ $=17570$. There is an inflection point in both the order parameters and the structure factor around $22 \mathrm{~K}$, which is the azimuthal angle disorder transition temperature. 


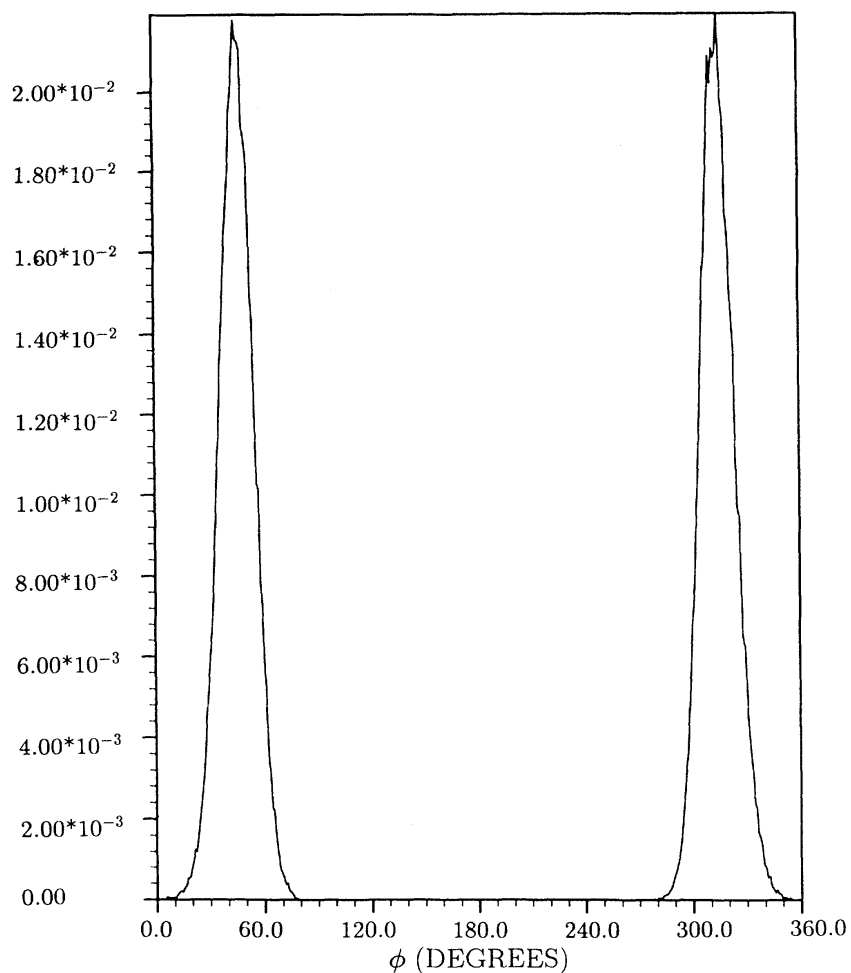

(a)

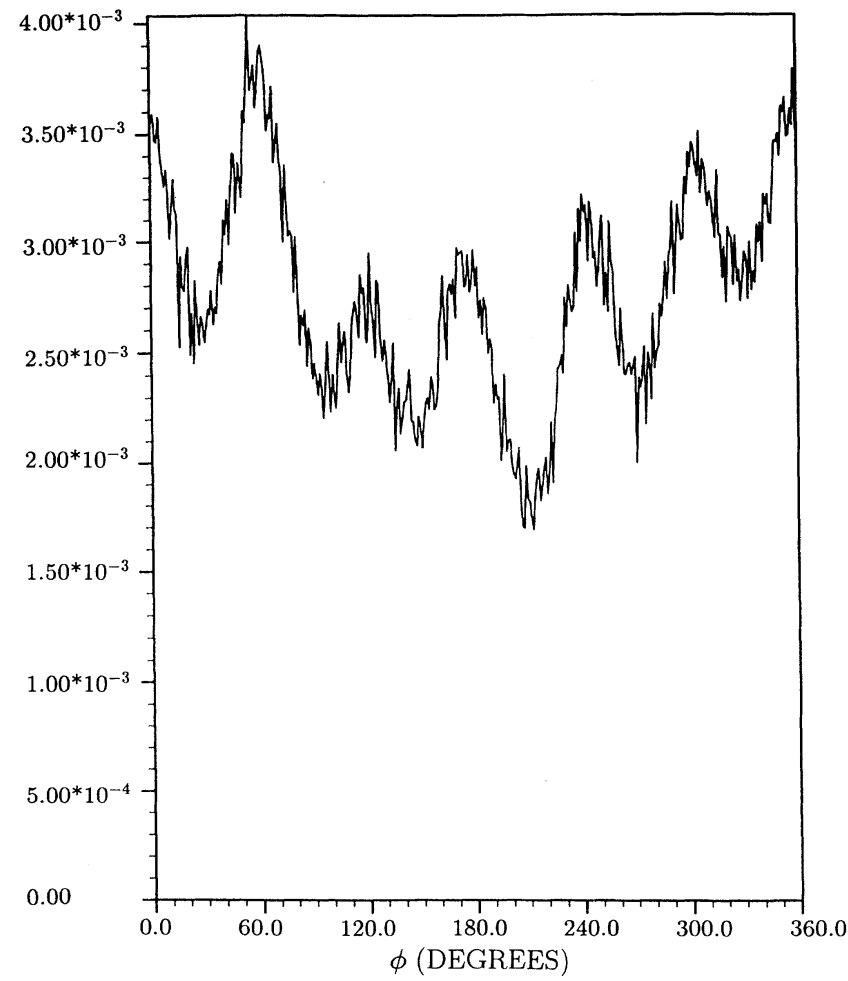

(c)

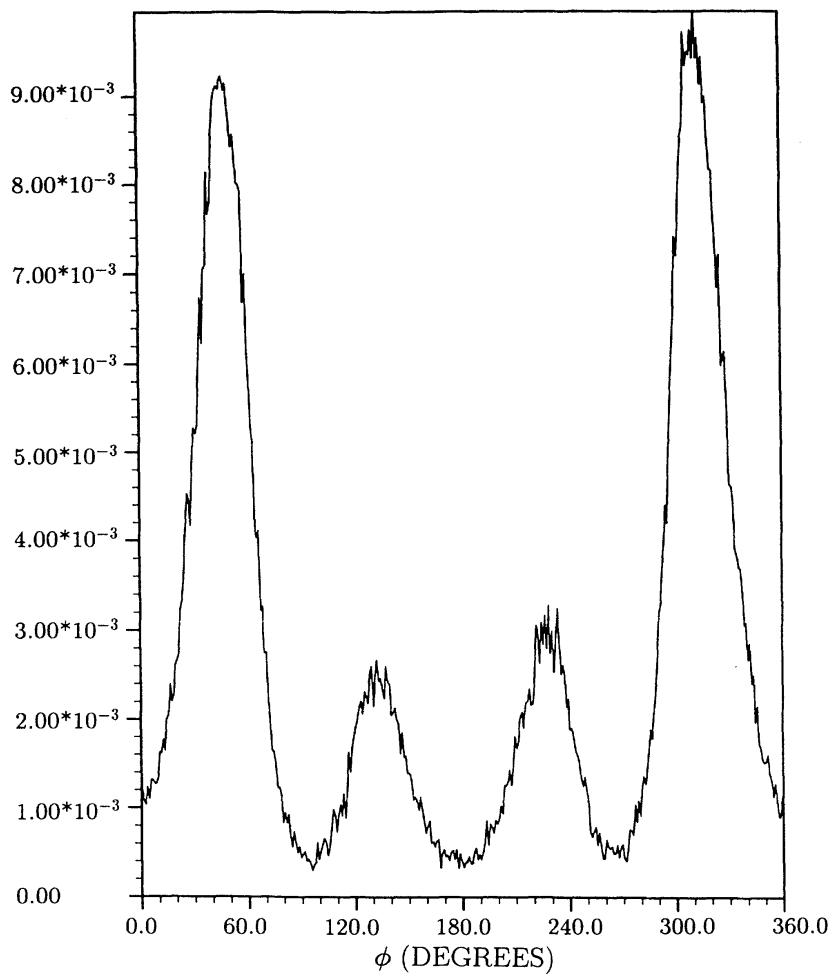

(b)

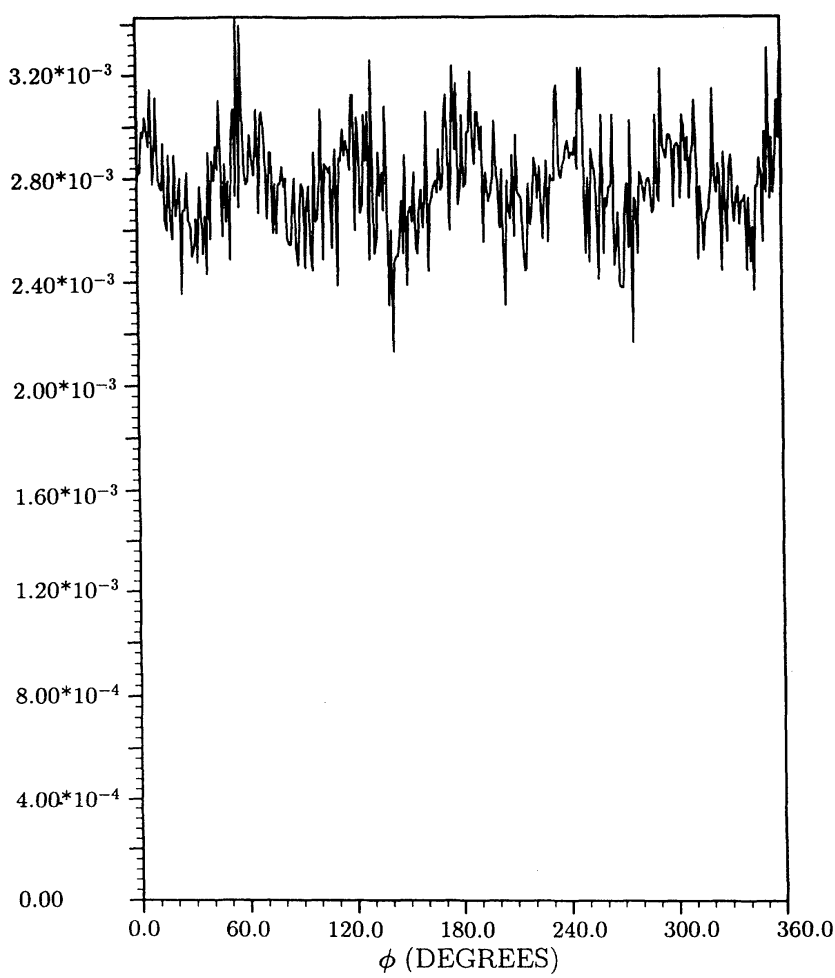

(d)

FIG. 2. The N-N azimuthal angle distribution as a function of the temperature. (a) $T=10 \mathrm{~K}\left(T / T_{\text {melt }}=0.137\right)$, well below the disorder transition at $22 \mathrm{~K}$. (b) $T=20 \mathrm{~K}\left(T / T_{\text {melt }}=0.274\right)$, just below the transition. (c) $T=25 \mathrm{~K}\left(T / T_{\text {melt }}=0.342\right)$, just above the transition. Notice the distinct sixfold structure in the distribution function. (d) $T=50 \mathrm{~K}\left(T / T_{\text {melt }}=0.685\right)$. The sixfold structure in the distribution function is barely visible at this temperature, so that the azimuthal motion evolves from a hindered rotation to a free rotation in this temperature range. 


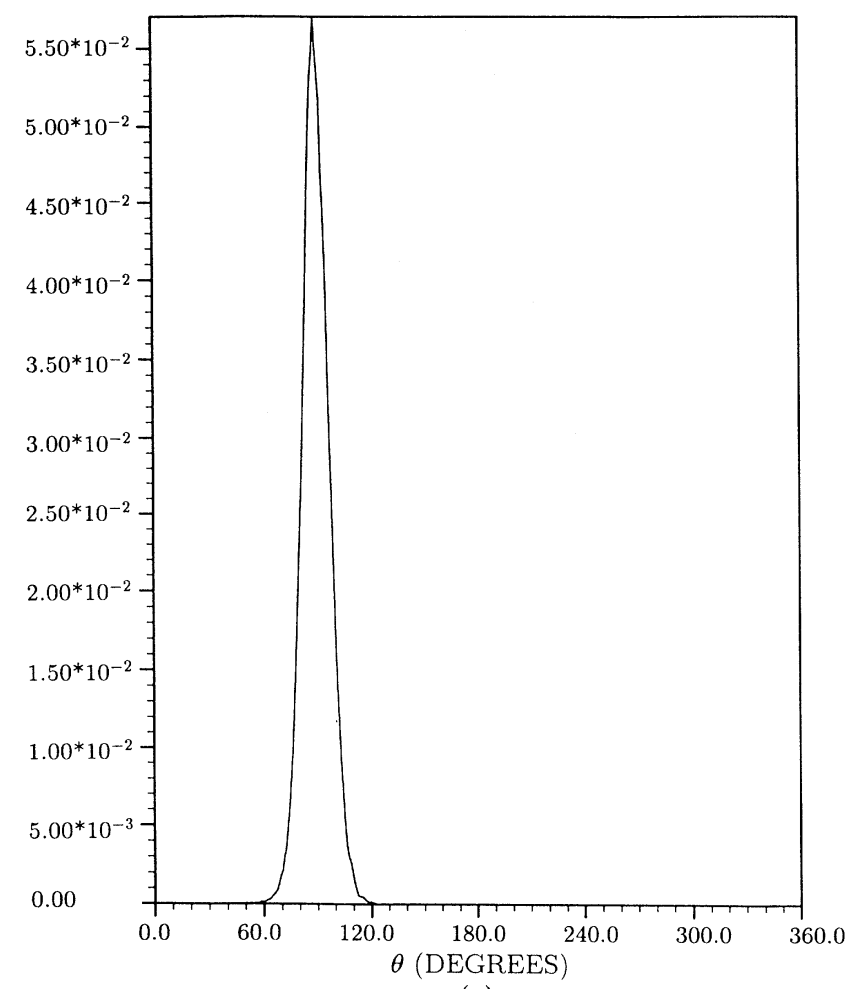

(a)

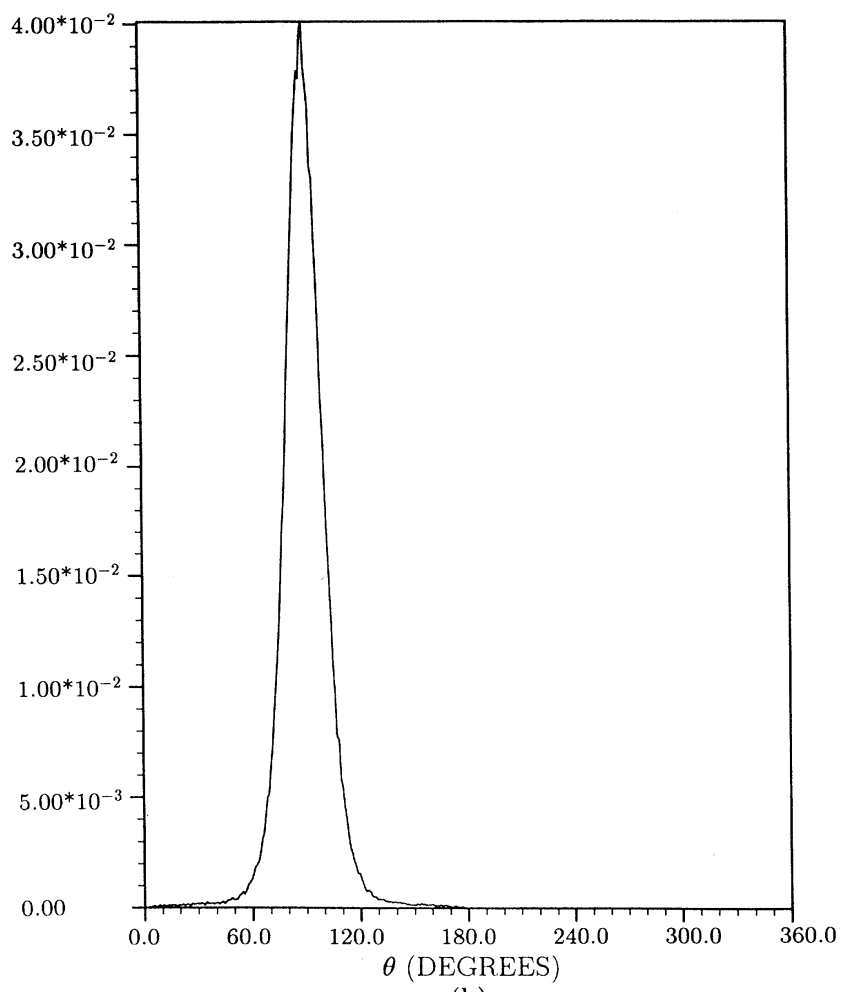

(b)

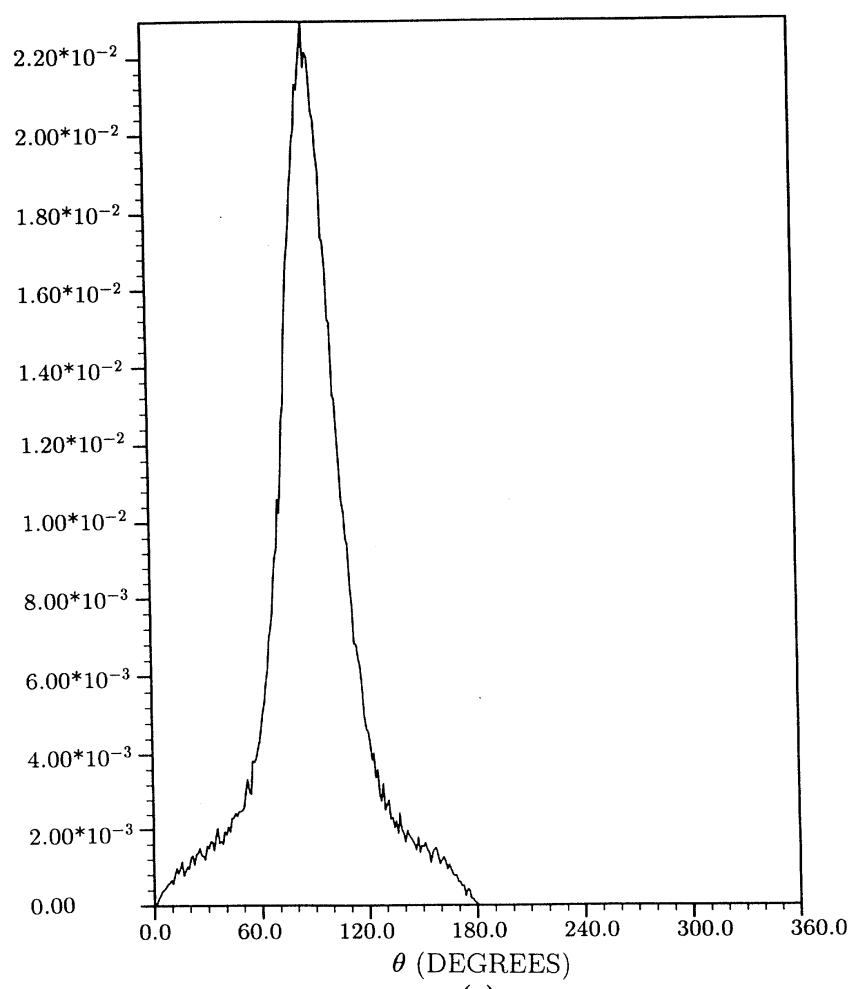

(c)

FIG. 3. The distribution of the N-N polar angle as a function of temperature. The polar angle is $90^{\circ}$ for the N-N bond parallel to the surface. (a) $T=15 \mathrm{~K}\left(T / T_{\text {melt }}=0.205\right)$, well below the azimuthal disorder transition temperature at $22 \mathrm{~K}$. (b) $T=25 \mathrm{~K}\left(T / T_{\text {melt }}=0.342\right)$, just above the transition. (c) $T=50 \mathrm{~K}\left(T / T_{\text {melt }}=0.685\right)$, well above the transition. 
in the azimuthal angle. Above the transition, there is a sixfold pattern in the distribution function which persists to $50 \mathrm{~K}$; it corresponds to a hindered rotation which reflects the sixfold symmetry of the graphite substrate. Only above $50 \mathrm{~K}$ does the distribution become nearly uniform, corresponding to a nearly free rotation. These characterizations of the motion are consistent with the average order parameters shown in Fig. 1 and with the

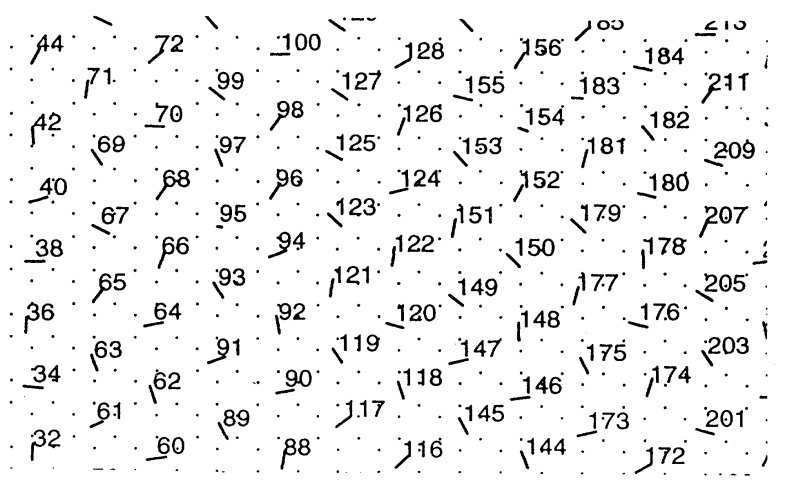

(a)

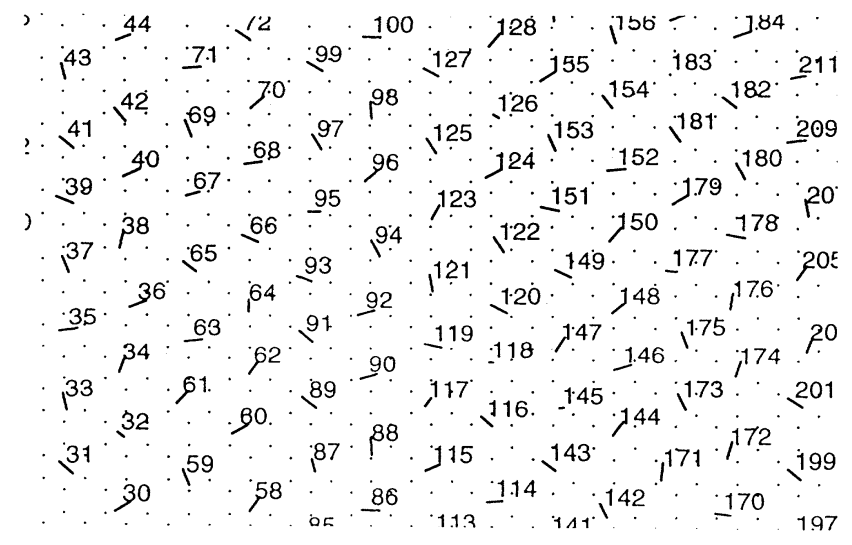

(b)

FIG. 4. Instantaneous configurations of the nitrogen molecules on the graphite surface at several temperatures. The small dots forming hexagons indicate the graphite carbon atom positions. The nitrogen molecules are shown as small lines which represent the projection of the $\mathrm{N}-\mathrm{N}$ bond on the $x y$ plane parallel to the graphite surface plane. The tilt of the nitrogen bonds with respect to the surface may thus be inferred from the length of the lines which degenerate into a dot for molecules with the bond perpendicular to the surface. (a) A section of an instantaneous configuration at $T=25 \mathrm{~K}$, just above the azimuthal disorder transition at 22 K. Notice the pinwheel structure element around molecule 95 , whose bond is almost perpendicular to the surface and to a lesser degree around molecules 154 and 183. (b) Same as in (a), but at $T=50 \mathrm{~K}$, well above the transition temperature. More molecules, such as $32,117,118,126,145$ and 177 , have their bonds tilted away from the surface plane and more or less "stand on end." There are pinwheel structure elements around these molecules. rotational correlation functions discussed in Sec. V.

There is a qualitative change in the distribution of the tilt $\theta$ angle of the $\mathrm{N}-\mathrm{N}$ axis on passing through the orientational disordering transition. As shown in Fig. 3, the distribution at low temperatures has the form of a one-component Gaussian distribution centered at $90^{\circ}$, which corresponds to molecular axes parallel to the surface. Above the transition, the distribution develops a second component and has a small very broad distribution superposed on the large narrow component. The narrow component reflects, as before, the librational motion of the molecules against the surface, whereas the broad component is a signature of molecules which are "standing on end," or nearly so. There are transient "pinwheel-like" structures in the solid, as evident in the instantaneous configurations at 25 and $50 \mathrm{~K}$ presented in Fig. 4. Figure 3 also shows that the two-component structure of the distribution becomes more pronounced with increasing temperature.

The hexagonal center of mass lattice melts at around 70-75 K. In Fig. 5, we show the intensity $S(1,1)$ of the leading Bragg reflection. It drops drastically in the temperature range of 70-75 $\mathrm{K}$ and from the position of the inflection point we infer a melting temperature of $73 \mathrm{~K}$. This value is a little lower than experimental results of $75-83 \mathrm{~K}$ (Ref. 36) and a value of $84 \mathrm{~K}$ in a Monte Carlo simulation. ${ }^{9}$ A possible reason for this will be discussed in section VI. The intermolecular potential energy per molecule as a function of temperature is also shown in Fig. 5; this energy increases sharply in the same temperature range as the drop in the $(1,1)$ reflection, with an inflection around $73 \mathrm{~K}$.

The atomic distribution function in the $z$ direction in

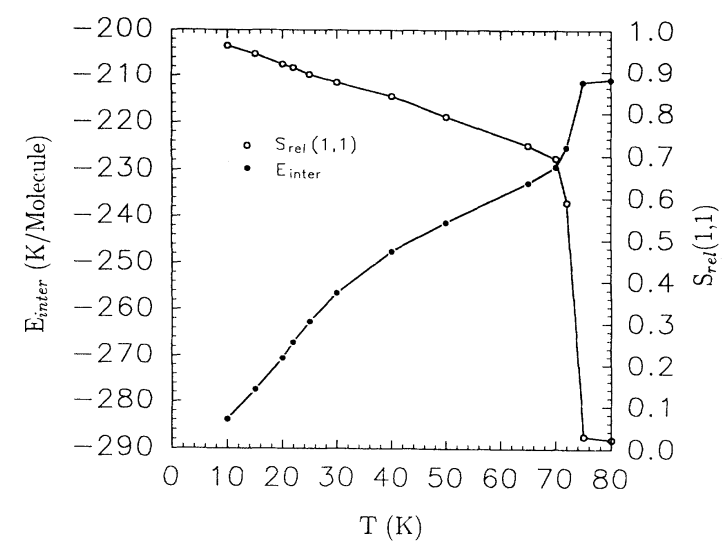

FIG. 5. Determination of the monolayer melting point. $S_{\text {rel }}(1,1)$ is the relative structure factor with Miller indices $(1,1)$ of the herringbone lattice with respect to the structure factor at $0 \mathrm{~K}$, which was determined to be 126862 . $S_{\text {rel }}(1,1)$ shows a strong drop in a temperature range of 70-75 K; from the inflection point, we assign the melting temperature as 73 $\mathrm{K}$. This is consistent with the plot of the intermolecular potential energy per molecule, $E_{\text {inter }}$, also shown here. It has a sharp variation in the same temperature region with an inflection point at $73 \mathrm{~K}$ and a weaker inflection near the azimuthal disorder temperature, around $22 \mathrm{~K}$. The latter transition is barely visible in the $S_{\text {rel }}(1,1)$ structure factor. 
Fig. 6 shows that there is no significant layer promotion of molecules until just a few degrees below the melting point. Then with further increase in temperature, molecules are promoted to a second layer, vacancies are created in the monolayer, and it melts. That is, the monolayer melting is associated with the formation of vacancies in the monolayer by promotion of molecules to a second layer. This observation agrees with the conclusions of Etters and co-workers ${ }^{9}$ about the melting mechanism.

The correlation between the change in polar angle distribution and the onset of disorder in the azimuthal angle reflects the fact that the molecule is somewhat ellipsoidal. By tilting a few molecules away from the surface enough space is created for the other molecules to disorder in the azimuthal angle. Constraining the molecules to move in the plane of the surface would probably not eliminate the orientational-disorder transition but shift it to a higher temperature. The melting point was shown in a simulation ${ }^{9}$ to increase by $13 \mathrm{~K}$ when the molecules are confined to the $x y$ plane; an increase in the orientational disorder temperature would also be expected. In monolayer films of ethylene, ${ }^{34}$ an orientational disorder transition has also been observed, but in films of butane and hexane no such transition exists; ${ }^{18}$ the aspect ratio of these molecules is simply too large. We expect that there is a crossover from films with an orientationaldisorder transition to films with none for molecules with an aspect ratio between that for nitrogen and ethylene and that for butane.

However, the almost spherical shape of the nitrogen molecule also means that the reduction of the "footprint" of the molecule on the surface by tilting is too small to create enough free area to initiate the melting process. Another mechanism is therefore necessary: here the promotion of molecules to a second layer is the energetically favorable way to create enough free surface area for the other molecules to translationally disorder. This is in contrast to what was observed in simulations for molecules with larger aspect ratios. ${ }^{18}$ For butane films, the tilting of the large-aspect-ratio molecule created enough free area to initiate the melting process. Hexane molecules have an even larger aspect ratio and there is a footprint-reduction mechanism in which molecules coil into a gauche conformation. When that mechanism is suppressed in a simulation, ${ }^{18}$ the increase

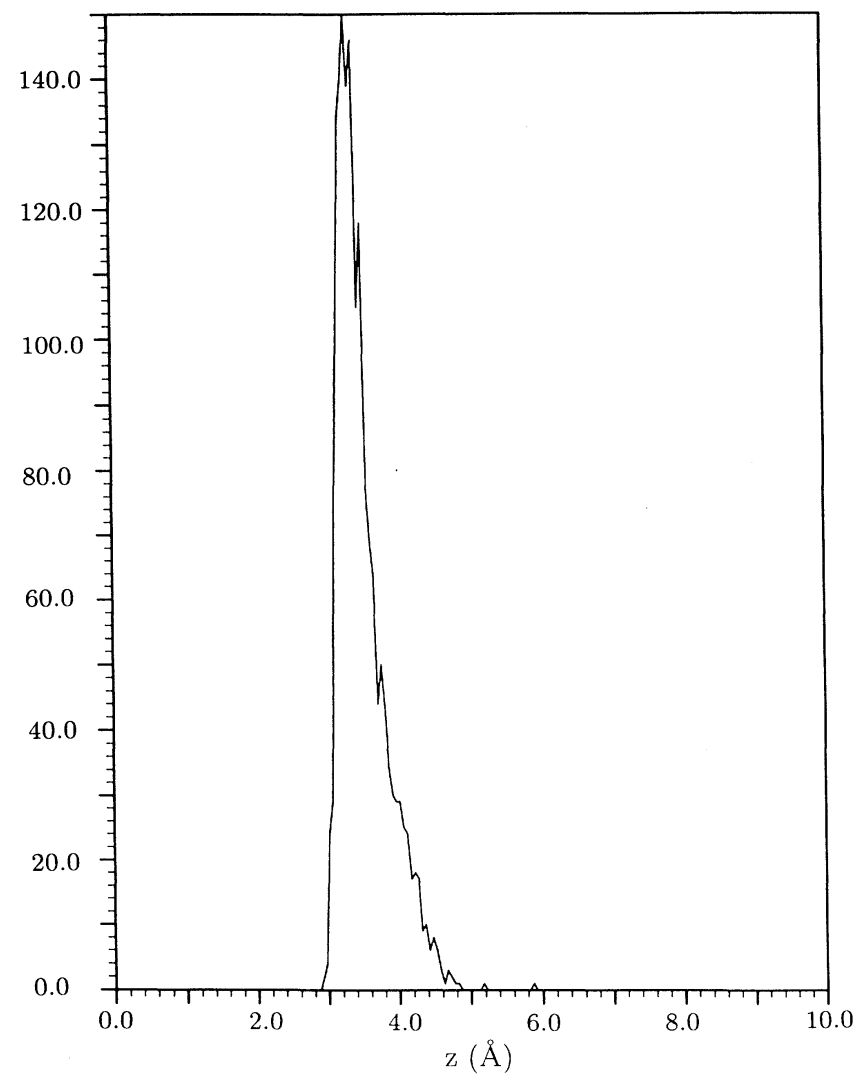

(a)

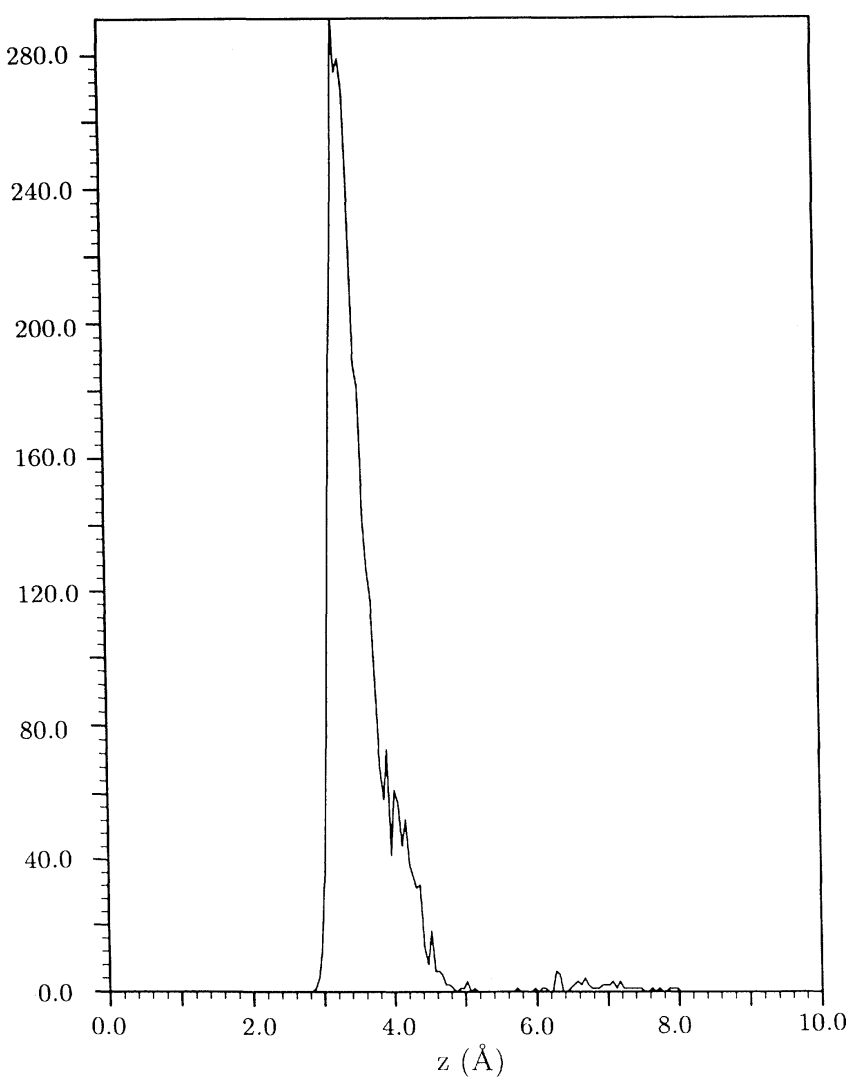

(b)

FIG. 6. The distribution of perpendicular distance of the $\mathrm{N}$ atoms from the surface; the second layer height is 6-7.5 $\AA$. (a) At $70 \mathrm{~K}\left(T / T_{\text {melt }}=0.959\right)$, just below the melting point at $73 \mathrm{~K}$, there are scarcely any molecules in the second layer. (b) At $72 \mathrm{~K}\left(T / T_{\text {melt }}=0.986\right)$, very close to the melting point, some molecules are now seen to have been promoted to a second layer. (c) At $75 \mathrm{~K}\left(T / T_{\text {melt }}=1.027\right)$ more molecules are promoted to a second layer and some desorb as seen from the small glitches in the distribution above $8 \AA$. 


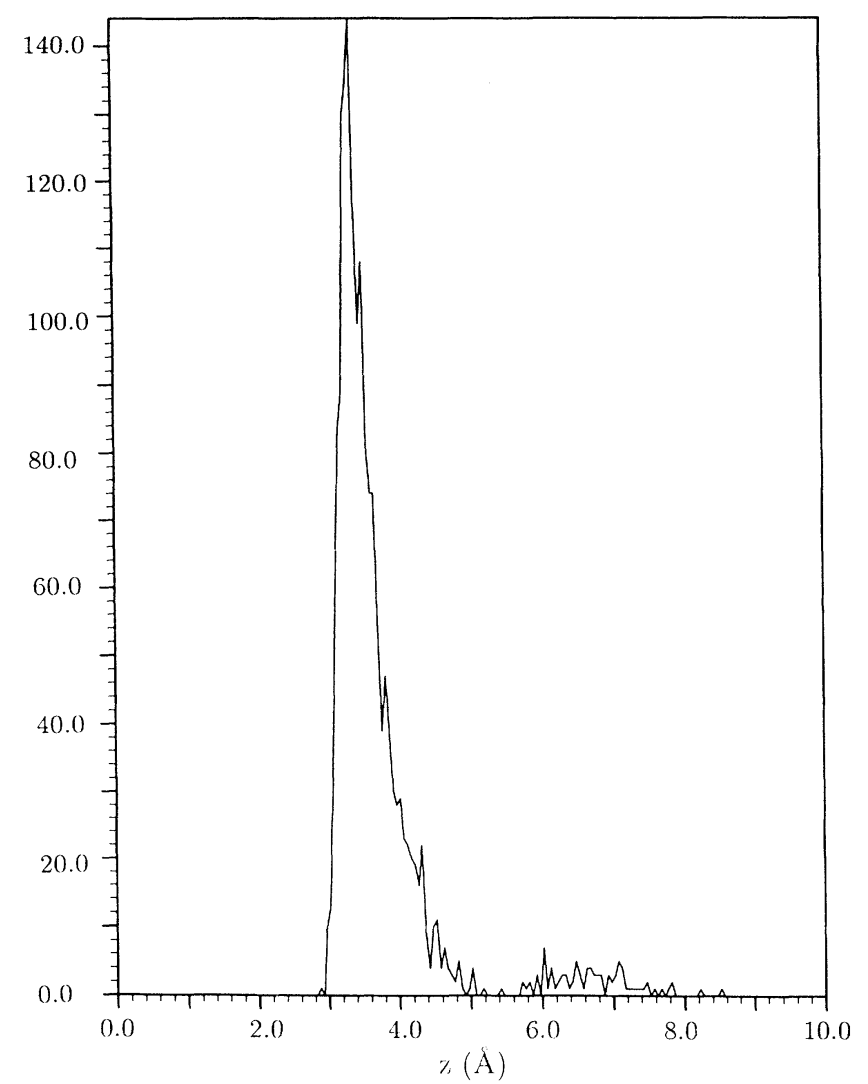

(c)

FIG. 6. (Continued).

in free area is accomplished by a combination of the processes described for the nitrogen films and butane films and the melting point is increased by $80-90 \mathrm{~K}$.

Figure 7 shows the temperature dependence of the average perpendicular distance $\bar{z}$ of the nitrogen molecules from the surface and of the average energy per molecule $E_{\text {cor }}$ arising from the corrugation terms in the holding potential. ${ }^{9}$ Since the corrugation amplitudes in Eqs. (23) and (29) vary exponentially with height $z$ (the leading dependence in the modified Bessel functions is also exponential), the temperature derivative of $E_{\text {cor }}$ is approximately

$$
\frac{d E_{\mathrm{cor}}}{d T} \simeq-g E_{\mathrm{cor}} \frac{d \bar{z}}{d T}
$$

where $\bar{z}$ is the average height of the center of mass of the molecules. The increase in the average height arises from the thermal average of independent molecular motions in the laterally averaged holding potential and from relaxation as the molecule moves laterally in the corrugated holding potential. The former effect is estimated with an auxiliary calculation of the Boltzmann average of the height of one molecule in the laterally averaged holding potential Eq. (22): the average slope with temperature from 10 to $60 \mathrm{~K}$ is $1.7 \times 10^{-3} \AA / \mathrm{K}$, which is $2 / 3$ of the slope shown for the molecular-dynamics average in Fig. 7. When it is used for the change in height for the

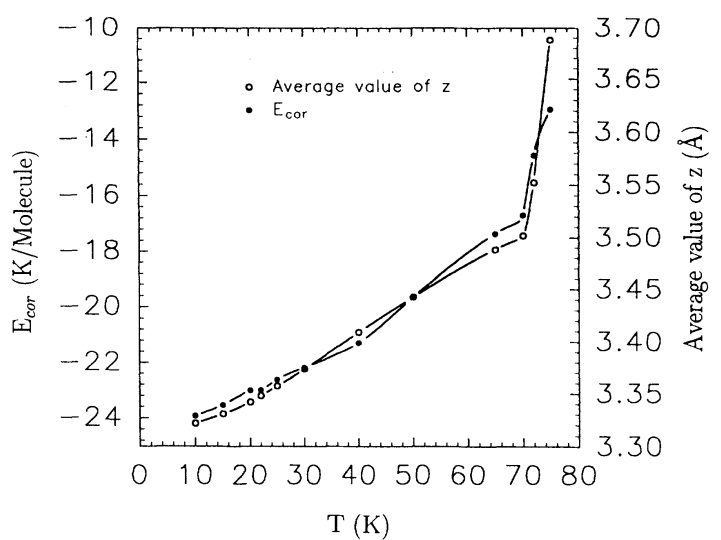

FIG. 7. The average corrugation energy per molecule $E_{\text {cor }}$ and the average distance $z$ of the molecules from the surface as a function of the temperature.

corrugation amplitude and with a value $E_{\text {cor }} \simeq-21 \mathrm{~K}$, Eq. (30) leads to an estimate $d E_{\text {cor }} / d T \simeq 0.10$, while the slope of $E_{\text {cor }}$ in Fig. 7 is approximately 0.11 .

The increase in $\bar{z}$ is rather smooth in the temperature range from 10 to $70 \mathrm{~K}$ and then it changes drastically. The change is correlated with the promotion of molecules to a second layer near the onset of melting. Several molecules desorb at temperatures of $75 \mathrm{~K}$ and above and there is a rapid increase in second layer occupation.

Even at the melting point, the average corrugation energy is about $15 \mathrm{~K} /$ molecule; thus the lateral motion of the molecules has the character of a hindered translation near the melting point. Since there is not a clear signature of the orientational disorder transition at $22 \mathrm{~K}$ in Fig. 7, this transition does not involve drastic changes in the average perpendicular distance or in the corrugation energy.

\section{DYNAMICAL PROPERTIES}

We start with a discussion of the mean square displacement of a molecule as a function of temperature, shown in Fig. 8. Below the melting point, each molecule oscillates about its equilibrium position above the center of a carbon hexagon in the graphite surface. The amplitude of the oscillation increases smoothly with temperature up to about $50 \mathrm{~K}$ and then it increases sharply to $5 \AA^{2}$ at $70 \mathrm{~K}$, just below the melting point. The distance from the center of a carbon hexagon in the graphite surface to a C-C bond is $1.23 \AA$, so that mean square displacements less than $\sim 3 \AA^{2}$ are consistent with molecular motions within a hexagon. The mean square displacements in Fig. 8 are consistent with such restricted motions, since the perpendicular motions are included in the mean square displacements and begin to contribute significantly at $70 \mathrm{~K}$.

The curve in Fig. 8(a) appears to diverge as the temperature approaches the melting point at $73 \mathrm{~K}$. At $75 \mathrm{~K}$, just above the melting point, the mean square displace- 


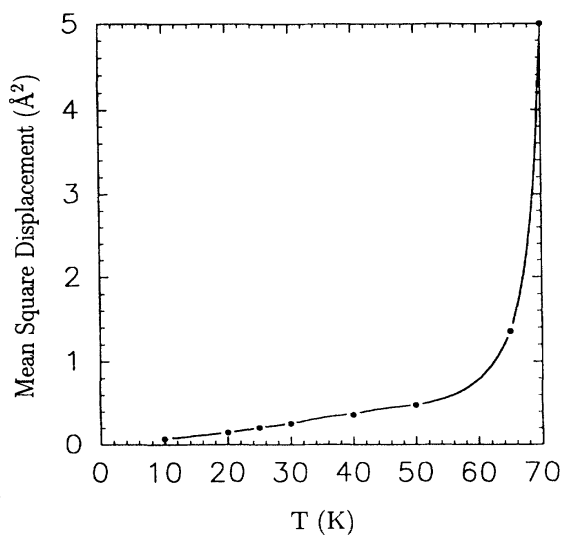

(a)

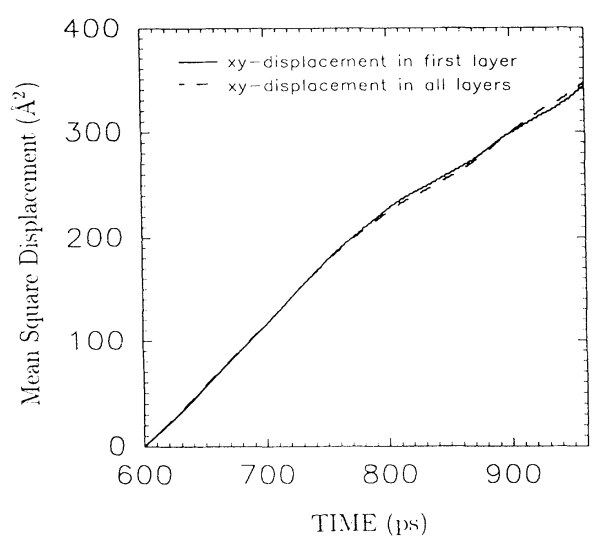

(b)

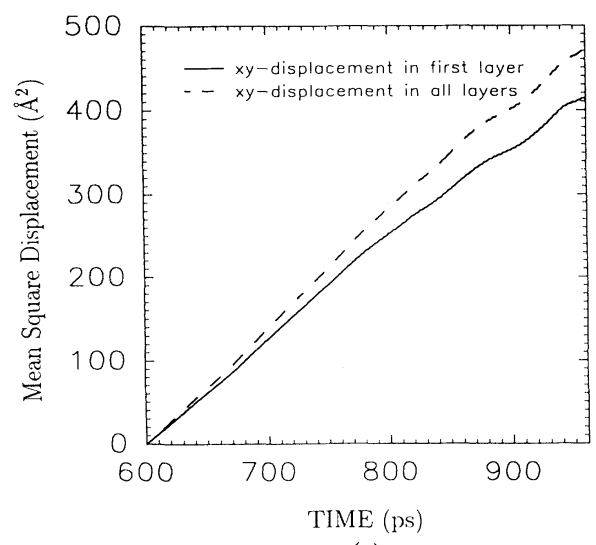

(c)

FIG. 8. Mean square displacement of the molecular center of mass (a) as a function of temperature for the solid below the melting point, (b) as a function of time for the fluid at 75 $K$, just above the melting point, and (c) as a function of time for the fluid at $80 \mathrm{~K}$. For (b) and (c) the initial time of the calculation is at $600 \mathrm{ps}$ in the total run. ment is no longer bounded. This is clear from Fig. 8(b), where the mean square displacement after $350 \mathrm{ps}$ is 340 $\AA^{2}$ and continues to grow with time. The change in slope at 800 ps elapsed time correlates with a small decrease in the second layer occupancy of the film; the evolution of the second layer occupancy of the film is not shown here. A similar but less pronounced change is observed in the calculation at $80 \mathrm{~K}$. The two-dimensional diffusion coefficient derived from the slope of the mean square displacement at $800-960 \mathrm{ps}$ is $1.8(2.8) \times 10^{-5} \mathrm{~cm}^{2} / \mathrm{sec}$ at 75 (80) K. The diffusion constant of a monolayer fluid of methane/graphite has a similar magnitude. ${ }^{39}$

The mean square displacements shown in Figs. 8(b) and $8(\mathrm{c})$ are calculated for "surface molecules." If a molecule once in the 400-ps time sequence leaves the surface and goes into the $3 \mathrm{D}$ gas phase, defined as the center of mass $z$ coordinate $>8 \AA$, it is completely dropped from the mean square displacement calculation. There are 48 (59) such molecules at $75 \mathrm{~K}(80 \mathrm{~K})$. Similarly, we identify molecules for calculation of the mean square displacement of first layer molecules by dropping molecules from the calculation if somewhere in the 400-ps time sequence they have had a center of mass $z$ coordinate $>6$ $\AA$; there is a total of 81 (98) such molecules at $75 \mathrm{~K}(80$ $\mathrm{K})$. The average number of molecules in the first layer at $75 \mathrm{~K}$ is 214 and in the first two layers 223 , where the perfect commensurate lattice had 224; the corresponding numbers at $80 \mathrm{~K}$ are 212 and 222 molecules. As shown in Figs. $8(\mathrm{~b})$ and $8(\mathrm{c})$, the distinction between mean square displacements calculated for only first-layer molecules and for molecules in both layers becomes significant at $80 \mathrm{~K}$. The diffusion constant in the second layer is expected to be larger than in the first layer and at $80 \mathrm{~K}$ there is a sufficient number of molecules in the second layer to make the displacement curves distinct. The curves may be modeled by assigning different diffusion constants for molecules in the first and second layers. A preliminary estimate for the $80 \mathrm{~K}$ data, using the average populations of the first and second layers to form a weighted average, is that the first layer diffusion constant is $\sim 2.4 \times 10^{-5} \mathrm{~cm}^{2} / \mathrm{s}$ and the second layer diffusion constant is $\sim 1.1 \times 10^{-4} \mathrm{~cm}^{2} / \mathrm{s}$.

In the following we present and discuss the thermal evolution of a series of correlation functions.

\section{A. Rotational and velocity correlation functions}

We start with the frequency spectra of the timecorrelation functions at $T / T_{\text {melt }}=0.14$ in Fig. 9. At this temperature the layer is a nearly harmonic solid. The bouncing mode along $z$ and the tilting mode in the polar angle $\theta$ have long correlation times of 13-15 ps and 5-7 ps, respectively, because these motions are primarily determined by the molecule-substrate interactions. The spectra of both motions in Fig. 9 are sharply peaked around $1.6 \mathrm{THz}$, so they may mix strongly as was seen in the lattice dynamics calculations for wave vectors away from the Brillouin-zone center. ${ }^{5,6}$ For single molecule motion in a perfect harmonic potential, the two spectra would be identical, but as seen from the superposition 
in Fig. 9(c), this is not quite the case. The translations in the $x$ and $y$ directions and the azimuthal rotation have more complicated autocorrelation functions, with correlation times of only 2-3 ps; they are strongly influenced by intermolecular interactions. Although there are normal modes with long lifetimes, the superposition of many

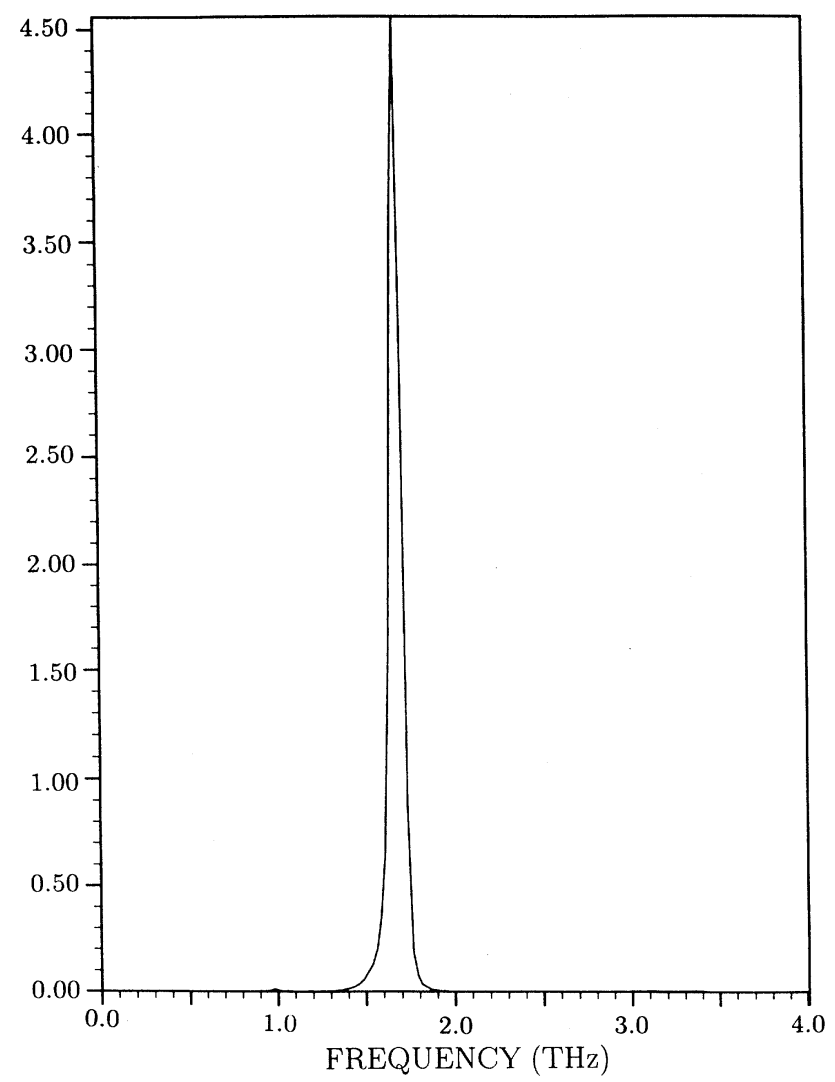

(a) normal modes results in time-correlation functions with a rapid decay. The spectra in Fig. 9 show three bands, near $0.5 \mathrm{THz}, 1.0 \mathrm{THz}$, and $1.5 \mathrm{THz}$. Thus there may be a strong mixing of azimuthal motions and lateral translations and a weaker mixing with the polar motion and perpendicular vibration. This is consistent with results

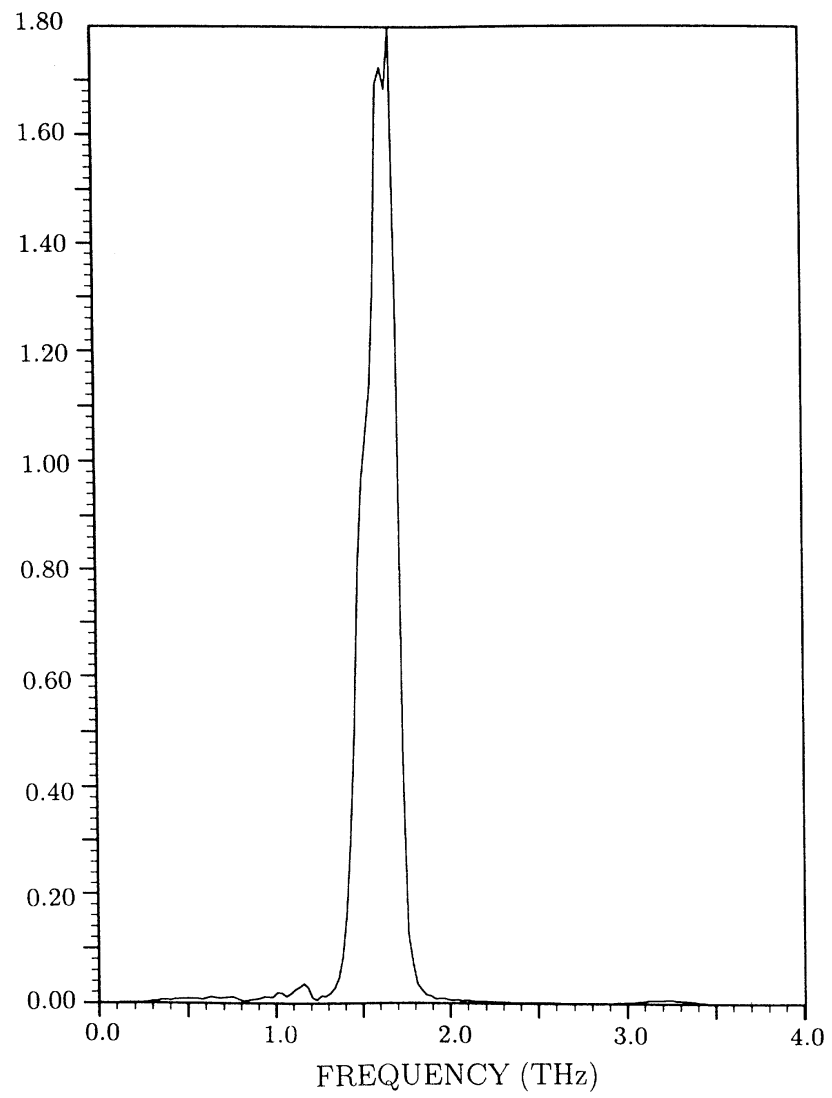

(b)

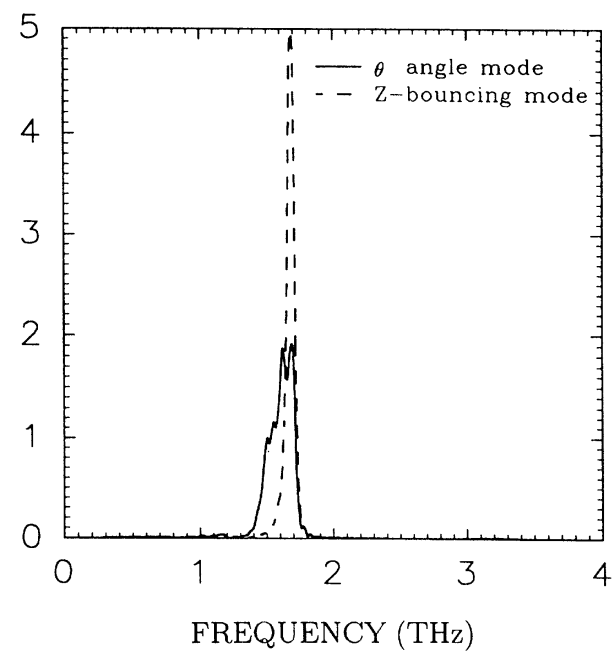

(c)

FIG. 9. Frequency spectra at $T=10 \mathrm{~K}\left(T / T_{\text {melt }}=0.137\right)$. (a) Translational motion of the molecules perpendicular to the surface, i.e., the bouncing mode. (b) The polar angle motion of the N-N bond, i.e., the tilting mode. (c) Superposition of the spectra for the tilting and bouncing modes. For single molecule motion in a perfect harmonic potential, the two spectra would be identical. (d) The lateral motion of the molecules in the $x$ direction. (e) The lateral motion of the molecules in the $y$ direction. (f) The azimuthal angle motion of the N-N bond. 


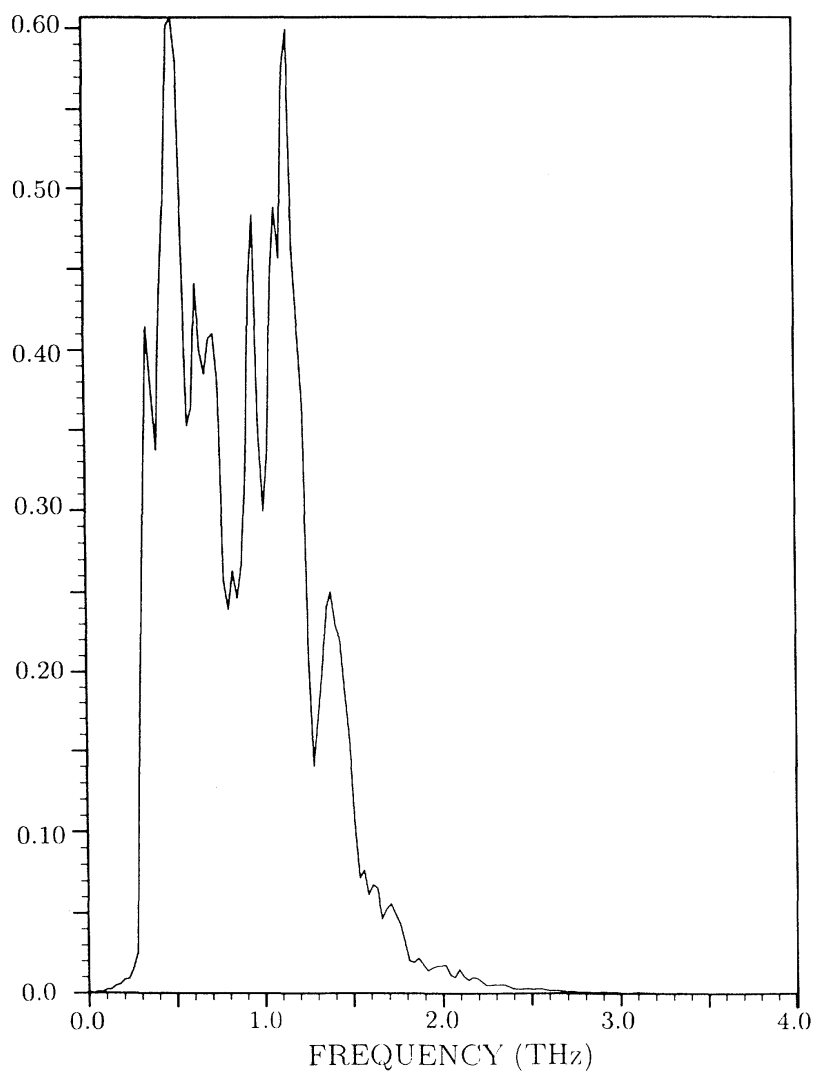

(d)

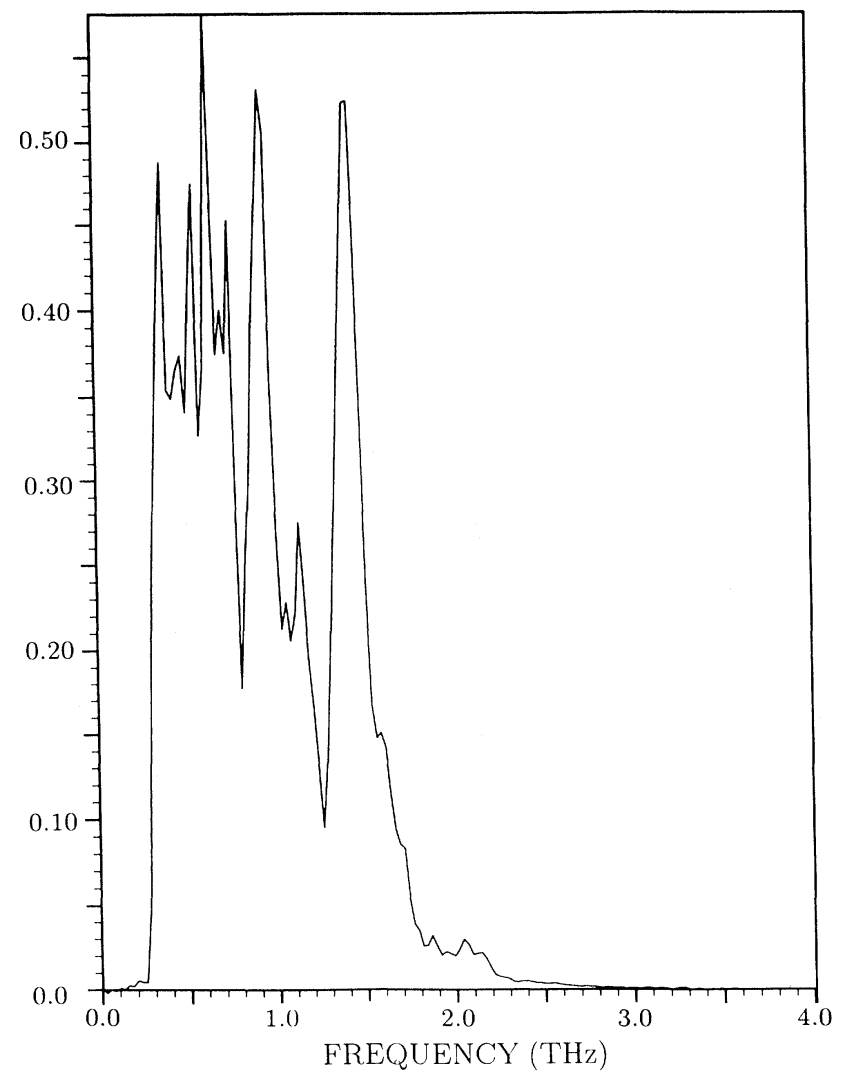

(e)

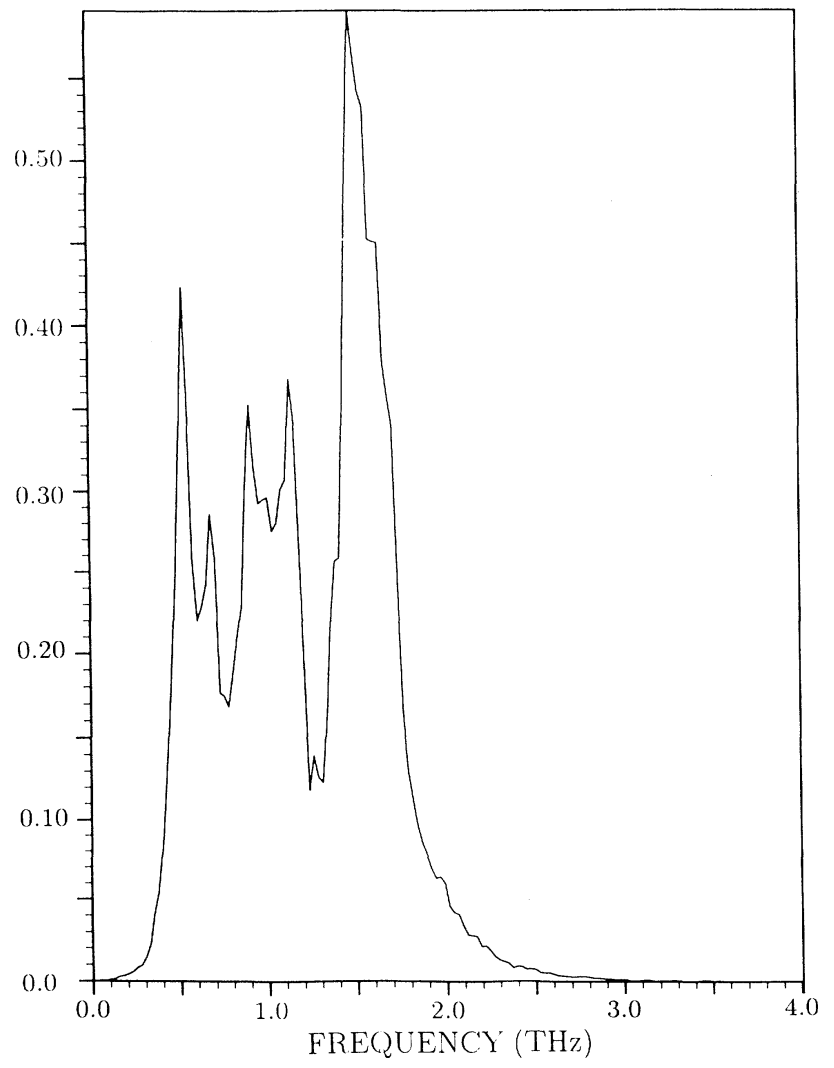

(f)

FIG. 9. (Continued). 


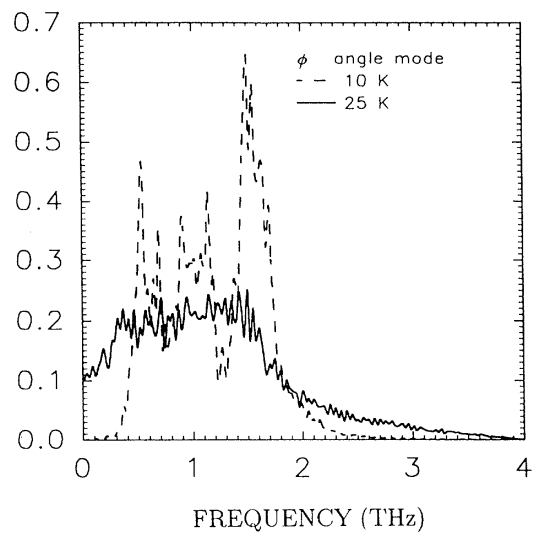

FIG. 10. Superposition of frequency spectra for the azimuthal angle motion at $T=10 \mathrm{~K}$, below the disorder transition temperature at $22 \mathrm{~K}$, and at $T=25 \mathrm{~K}$, just above the transition temperature. The low temperature spectrum is typical of a librational motion and the high temperature spectrum is typical of a hindered rotor motion with both diffusive and librational features.

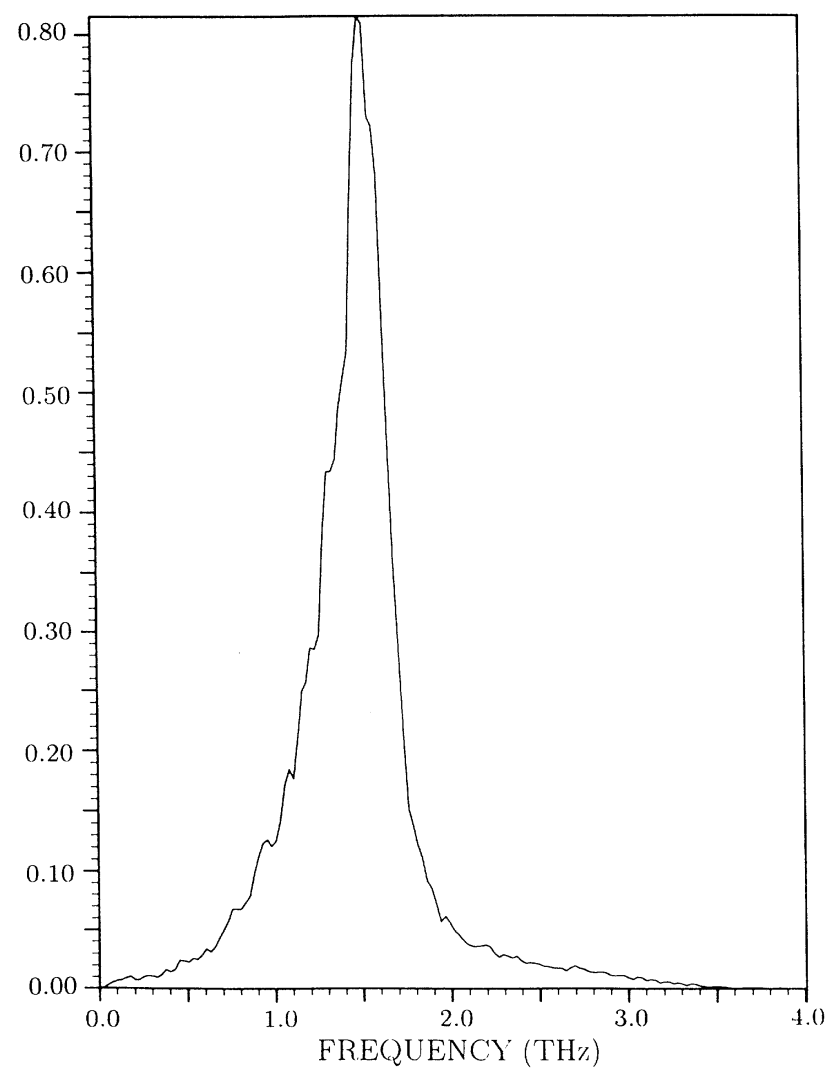

(a) of lattice dynamics calculations. ${ }^{5,6}$

Figure 10 shows that there is a prominent signature of the orientational disorder transition in the frequency spectrum of the azimuthal correlation function at $25 \mathrm{~K}$, which is just above the transition for our model. In addition to a broad peak near $1 \mathrm{THz}$, a finite spectral density persists to very low frequencies. The existence of a finite spectral density at zero frequency implies ${ }^{23}$ that there is some diffusional character to the motion, even if it is not totally random. The peak at $1 \mathrm{THz}$ shows that the rotor performs librational motion between diffusive steps, as expected for a hindered rotor.

At $T / T_{\text {melt }}=0.685$ in Fig. 11, the correlation times for the tilt angle mode and the bouncing mode have been reduced to 1-2 ps and 3-4 ps, respectively. In the spectra of the bouncing mode and the tilt angle mode, a broad low frequency shoulder is seen. As discussed below, this shoulder is associated with the tendency of molecules to tilt away from the surface when the temperature is raised. The frequency spectrum of the azimuthal angle motion is now more like a free diffusive rotational motion. The spectra for the $x$ and $y$ motions are quite similar to those

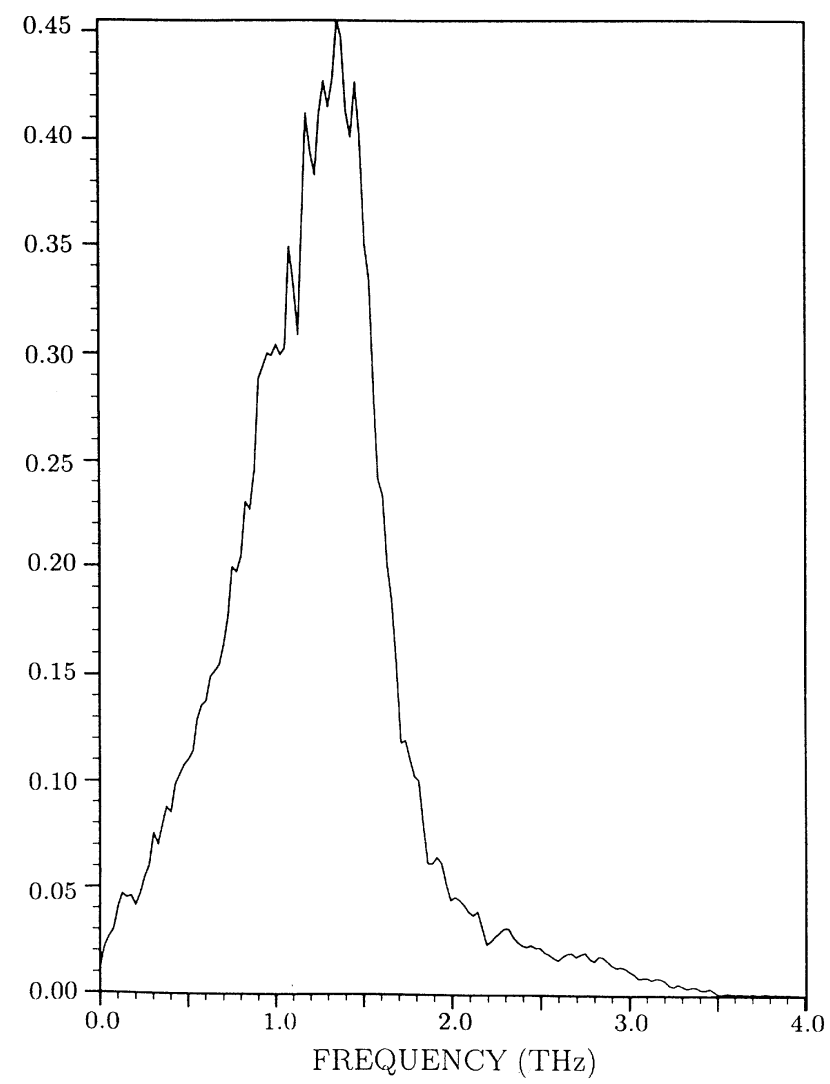

(b)

FIG. 11. Frequency spectra at $T=50 \mathrm{~K}\left(T / T_{\text {melt }}=0.685\right)$. (a) Translational motion of the molecules perpendicular to the surface, i.e., the bouncing mode. (b) The polar angle motion of the N-N bond, i.e., the tilting mode. (c) The lateral motion of the molecules in the $x$ direction. (d) The lateral motion of the molecules in the $y$ direction. (e) The azimuthal angle motion of the N-N bond. 


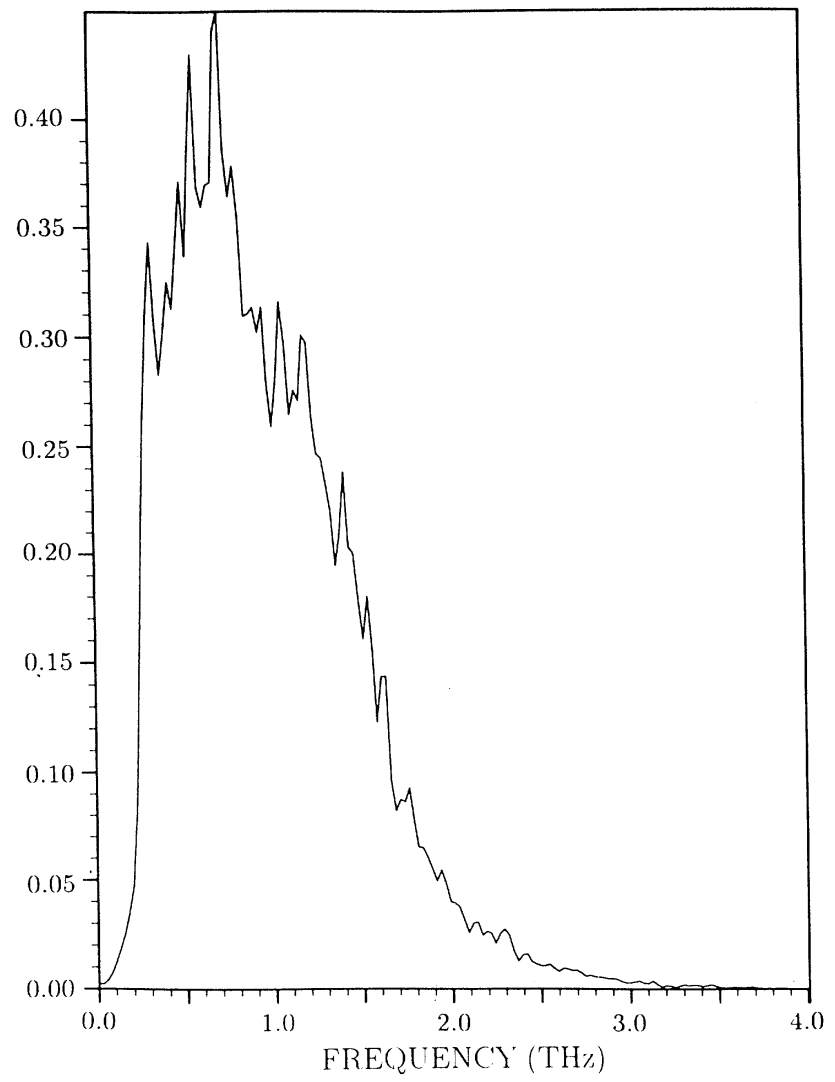

(c)

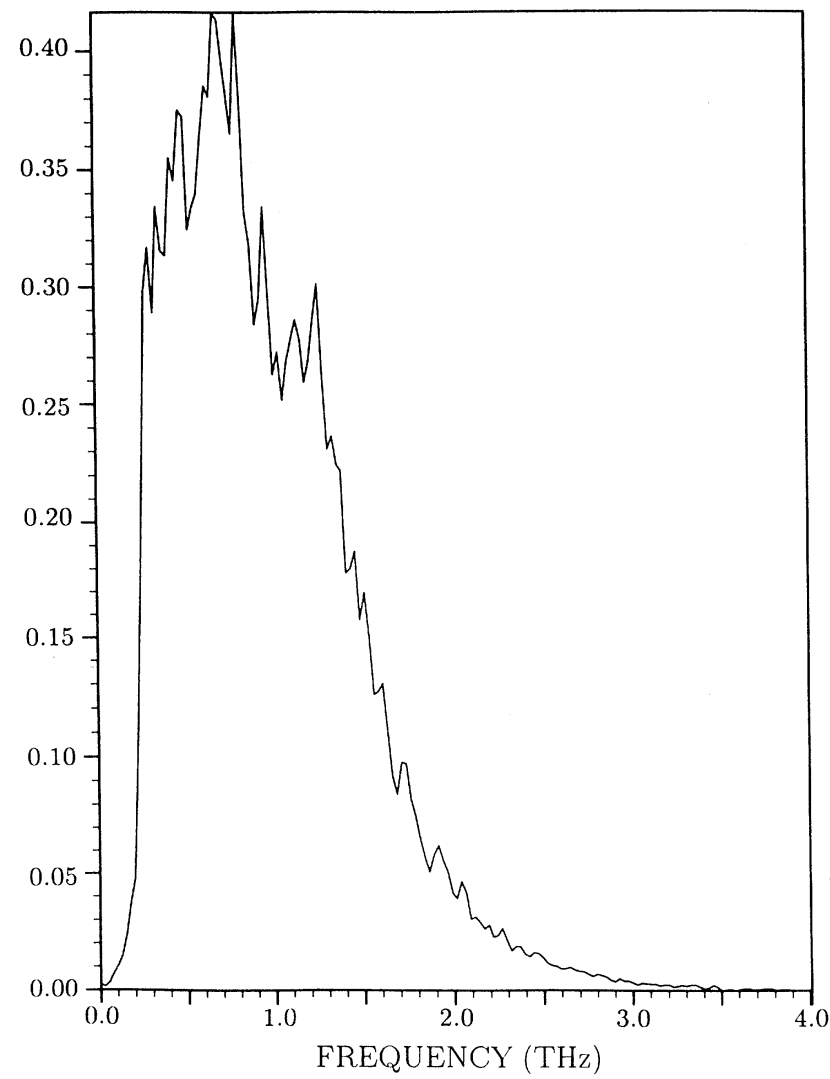

(d)

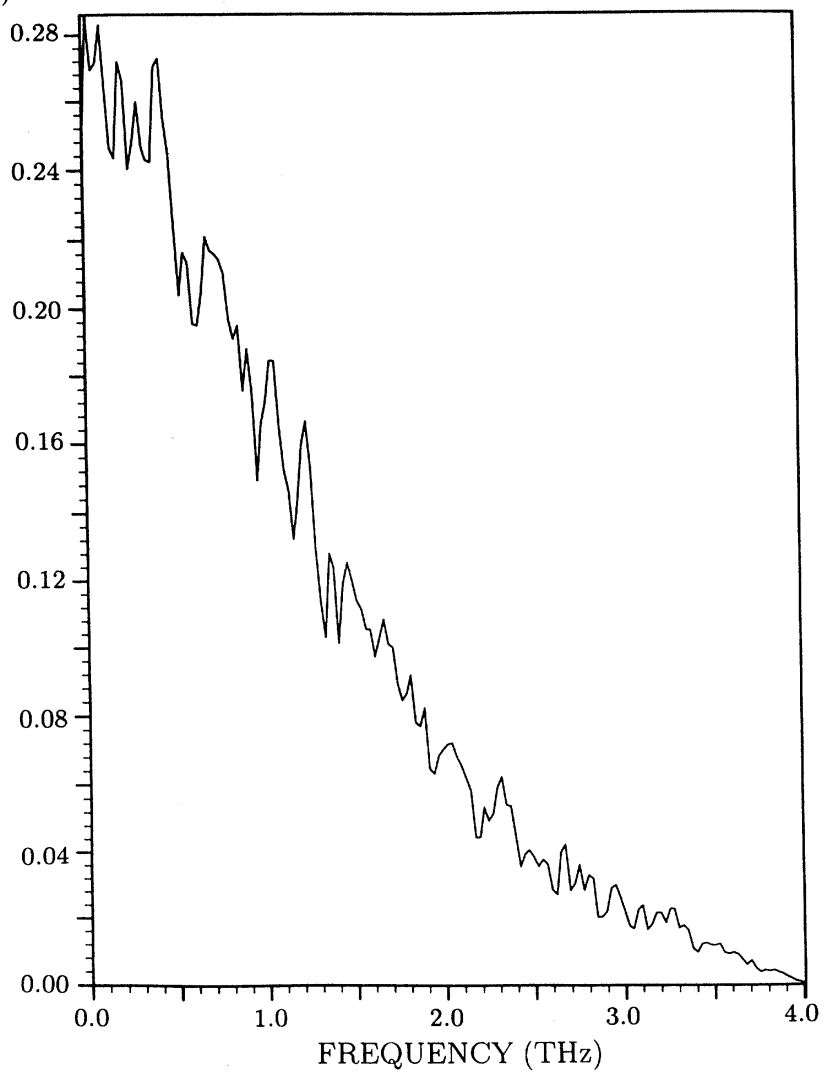

(e)

FIG. 11. (Continued). 
at low temperature, except for being broader and featureless.

A correlation between the orientational-disorder transition and the tendency to form pinwheel structural elements in the monolayer film is also seen in the frequency spectrum of the tilt angle correlation function. A broad low frequency component in the spectrum emerges above the transition temperature and becomes more prominent at higher temperatures, as shown in Fig. 12. The extra spectral density is attributed to molecules which are tilted away from the surface, i.e., stand on end, and have lower librational frequencies which are primarily determined by the neighboring molecules rather than by the substrate interaction. Superposed on the tilt angle spectra are the spectra of the perpendicular (bouncing) motion. These spectra also show a low frequency shoulder with increasing temperature, consistent with molecules tilted away from the surface, but the effect is less than that for the tilt angle motion because molecule-molecule

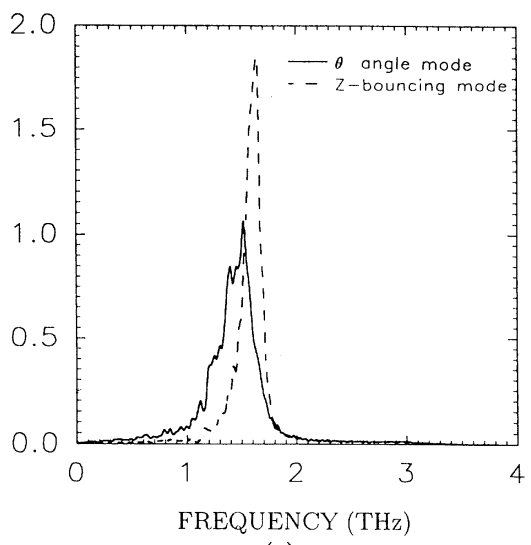

(a)

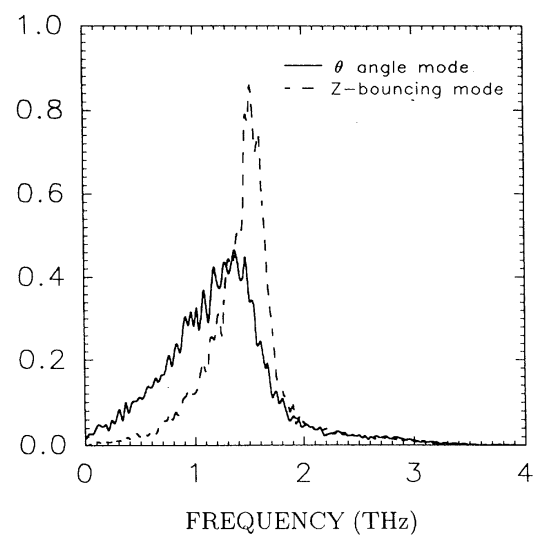

(b)

FIG. 12. Superposition of frequency spectra for the polar angle motion (tilt angle mode) and the translational bouncing mode perpendicular to the surface at (a) $T=25 \mathrm{~K}$, just above the azimuthal angle disorder transition at $22 \mathrm{~K}$, and (b) $T=50 \mathrm{~K}$. The emergent shoulders at low frequencies in both spectra are the signature of the existence of pinwheel structure elements in the monolayer film. interactions have a larger effect in the spectrum of the tilt angle motions.

\section{B. Intermediate scattering functions and the frequency gap}

We have calculated the modified intermediate scattering functions $I_{\text {coh }}^{\prime}(\kappa, t)$ and dynamical structure factors $S_{\text {coh }}^{\prime}(\kappa, \omega)$, defined in Sec. II B, at several temperatures and wave vectors. One goal of the calculations was to determine the temperature dependence of the frequency gap in the spectrum of acoustic modes, which arises at the center of the Brillouin zone of a commensurate monolayer solid. The scattering functions have therefore been evaluated at two reciprocal lattice vectors $1.703 \AA^{-1} \hat{\mathbf{x}}$ and $2.95 \AA^{-1} \hat{\mathbf{y}}$. With these wave vectors, the translational modes along $x$ and $y$ are probed at the Brillouinzone center and a test of the anisotropy of the response can be made. Both center of mass and atomic forms for the scattering functions were studied, but we show only results for the scattering functions evaluated with the molecular center of mass and the one-phonon approximations. Results for the other wave vectors show dispersion of the peak corresponding to the zone-center gap and have more structure. Results using atomic coordinates and the full scattering function do not differ much from those for the center of mass and one-phonon simplifications.

The results of calculations at $T=10 \mathrm{~K}\left(T / T_{\text {melt }}=\right.$ $0.137)$ are shown in Fig. 13. The intermediate scattering function $I_{\text {coh }}^{\prime}(\kappa, t)$, in the one-phonon-approximation, is shown at the two reciprocal lattice vectors. There is a well-defined oscillatory pattern with little damping and the Fourier transform has a single strong peak which gives the gap frequency for translational motion along the $x$ axis, [Fig. 13(a)] and along the $y$ axis, [Fig. 13(b)]. The spectra of the complete scattering functions, not shown here, are a little more noisy than the one-phonon spectra. The lifetime of the mode is much longer than the 20-ps window used in the calculations, as expected for a low temperature, nearly harmonic solid. The gaps for motion along the $x$ axis and along the $y$ axis are nearly equal, as found in lattice dynamics calculations.

In Fig. 14 we show the same pair of functions as in Fig. 13, but now for a temperature of $70 \mathrm{~K}\left(T / T_{\text {melt }}=\right.$ $0.959)$. The intermediate scattering function still is dominated by a well-defined oscillatory pattern, but the period of the oscillation is distinctly longer than for $10 \mathrm{~K}$. Although the temperature is close to the melting temperature, the phonon mode associated with the gap frequency is remarkably well defined as may be seen from the width of the main peak in the corresponding frequency spectra. It is only slightly broader than the corresponding low temperature peak and the increase is smaller than the frequency resolution of our MD calculation.

In Fig. 15 we show the gap frequencies $f(T)$ relative to the gap frequency at $T=0 \mathrm{~K}$ as a function of the temperature. For the zero temperature value, we use our lattice dynamics result for this interaction model $f(0)=0.30$ $\mathrm{THz}$; for the experimental data the value is $f(0)=0.40$ 
THz. The gap frequencies along the $x$ and $y$ axes are practically identical over the whole temperature range. The agreement with experimental data is quite good, although there is some uncertainty in comparing to the experimental data, because of uncertainty about the coverage in the experiments. ${ }^{4}$ The relative structure factor $S_{\text {rel }}(1,1)$ for the leading Bragg reflection of the center of mass lattice is also shown. It follows the gap frequency curve very well and there is no sign of a "floating solid" 38 with a zero frequency gap but a strong Bragg reflection. The decrease of the gap frequency with increasing temperature is the result of a complicated interplay between various factors. One factor is the anharmonicity of the corrugation. As the temperature is raised, anharmonic parts of the potential are probed by lateral motions of the molecules, with a softening of the gap frequency. Another major factor is the reduction in the amplitude of the corrugation by $\sim 30 \%$ from thermal expansion of the overlayer height, shown in Fig. 7. Both factors lead to a reduction in the sharpness of the commensurate lattice and are reflected in $S_{\mathrm{rel}}(1,1)$ : this is probably the reason that there is such a strong correlation between the two functions.

In summary, the gap frequency was determined as a function of increasing temperature until close to the melting temperature. The frequencies determined from the total scattering function and the one-phonon approximation are nearly equal. While the effect of multiphonon processes increases at higher temperatures, their effect near the melting temperature of this model remains too small for us to give a quantitative measure of the contributions. The gap frequencies determined for two reciprocal lattice vectors, which probed translations along perpendicular axes, were indistinguishable.

\section{CONCLUSIONS}

We have shown that our corrugation model, ${ }^{6}$ which includes electrostatic fields from aspherical charge distributions at atomic sites in the graphite crystal, leads to Brillouin-zone-center gap frequencies as a function of temperature which are in rather good agreement with experiments. For $\mathrm{N}_{2}$ /graphite a combination of van der Waals terms and electrostatic moment energies accounts for most of the corrugation for temperatures from $0 \mathrm{~K}$ to the melting point.

The zone-center frequency gap for the commensurate monolayer solid of $\mathrm{N}_{2}$ /graphite is found to be $19 \mathrm{~K}$. This is twice the value inferred for the gap of commensurate krypton/graphite from the analysis of specific heat experiments, so that it is a puzzle that there is not a

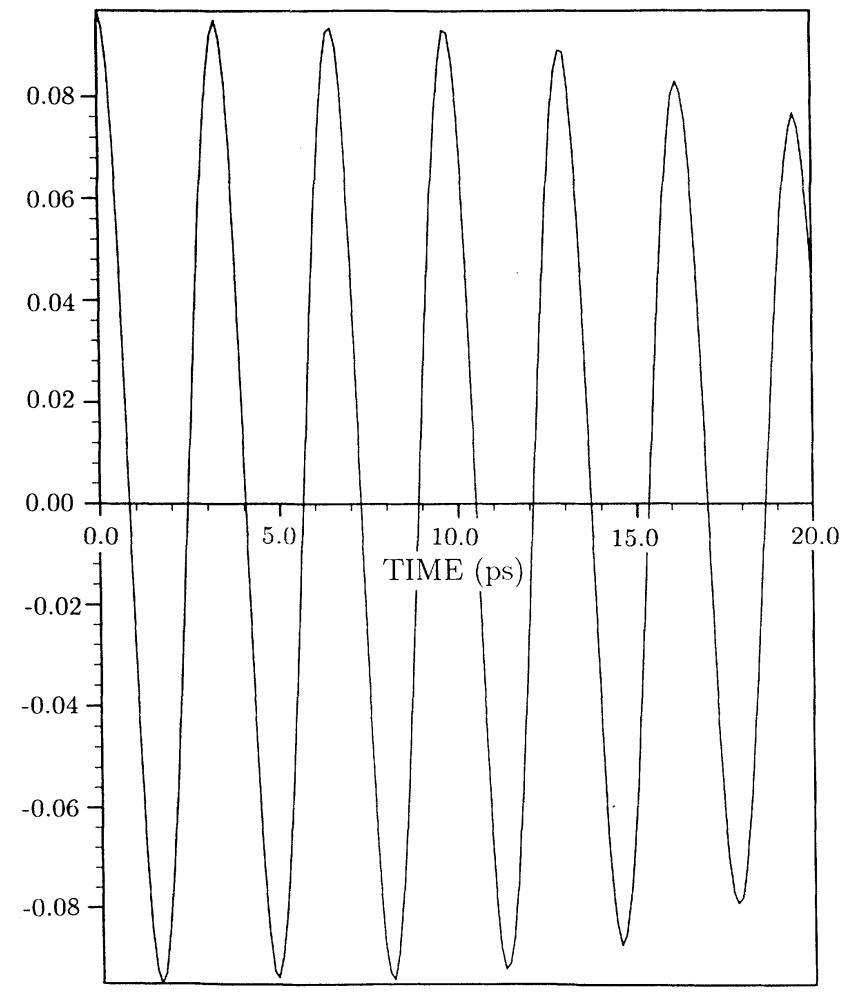

(a)

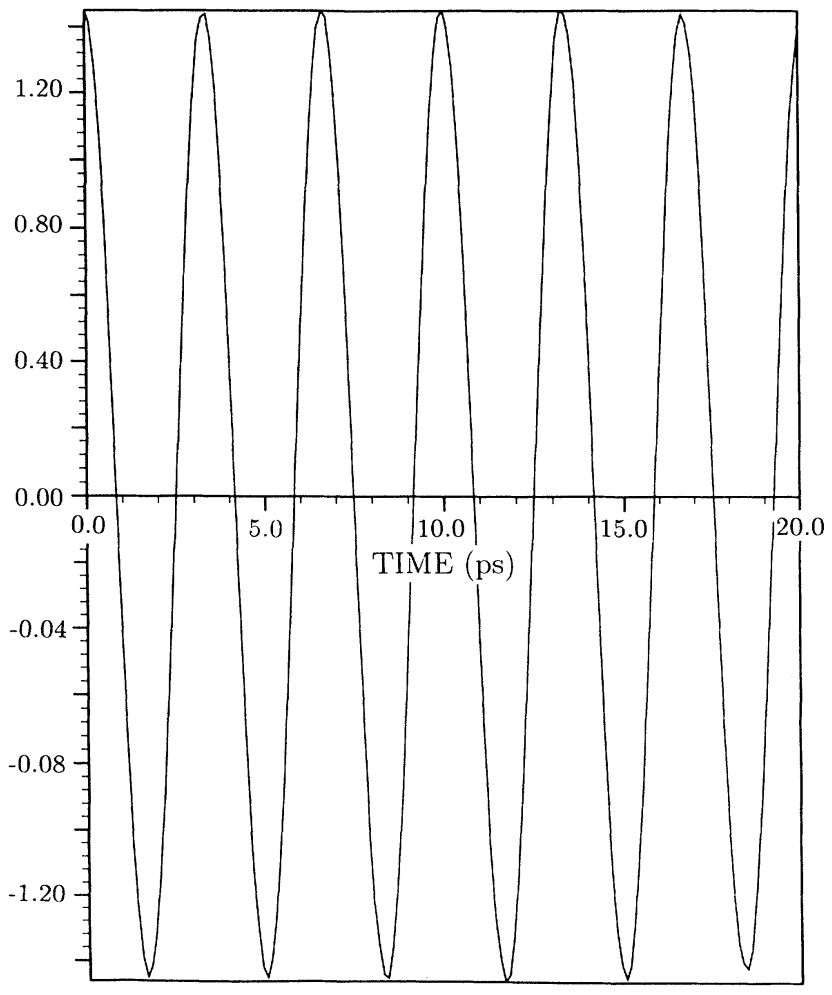

(b)

FIG. 13. One-phonon approximation to the intermediate coherent scattering function $I_{\text {coh }}^{\prime}(\kappa, t)$, for the center of mass motion at two reciprocal lattice vectors of the herringbone lattice and temperature $T=10 \mathrm{~K}\left(T / T_{\text {melt }}=0.137\right)$, (a) at the wave vector $\kappa=1.703 \AA^{-1} \hat{\mathbf{x}}$, which probes motion along $\hat{\mathbf{x}}$ at the Brillouin zone center and (b) at the wave vector $\kappa=2.95 \AA^{-1} \hat{\mathbf{y}}$, which probes motion along $\hat{\mathbf{y}}$ at the Brillouin zone center. 
prominent excitation gap in the corresponding specific heat measurements of the nitrogen monolayer. ${ }^{37}$

It was found that anharmonic effects of motion in the monolayer plane are only part of the mechanism for the decrease of the gap frequency with temperature. A major factor is the reduction in amplitude of the corrugation caused by an outward expansion of the $\mathrm{N}_{2}$ monolayer with temperature.

The marked effect of the sign of the surface quadrupole moment on the gap frequency has been shown again in the MD simulations; there are fewer structural constraints here than in our previous potential energy minimzations, but the effects remain quite similar. A positive quadrupole, as deduced from x-ray measurements of the charge density ${ }^{6}$ enhances the corrugation and improves the agreement with data for the gap frequency. A negative quadrupole moment, as determined by Vernov and Steele ${ }^{7}$ and Whitehouse and Buckingham, ${ }^{33}$ reduces the corrugation, changes the symmetry of the low temperature monolayer solid, and makes the agreement with experiments poorer.

A disorder transition in the azimuthal angle of the $\mathrm{N}$ $\mathrm{N}$ bonds was found at $T=22 \mathrm{~K}$. The transition is correlated with a change in the distribution of tilt (polar) angles and with changes in the Fourier spectra of the time-correlation functions of the polar angle and of the bouncing ( $z$-displacement) motions. In the instantaneous configurations, as the temperature increases, there is an increasing number of molecules with the $\mathrm{N}-\mathrm{N}$ axis nearly perpendicular to the surface. Since the aspect ratio of the molecule is slightly larger than one, the tilted molecules create space on the surface for the azimuthal orientations of other molecules to disorder when sufficient free space has been created.

The orientational disorder transition of the monolayer and the melting fall into a systematic pattern when viewed in the context of other linear or rod-shaped molecules. ${ }^{18,34}$ The aspect ratio of $\mathrm{N}_{2}$ is close to one and so the orientational-disorder transition occurs at a temperature far below the melting temperature. Also, this small aspect ratio accounts for the mechanism of melting, where molecules are promoted to a second layer in order to create enough free area in the first layer for the monolayer to melt. For systems of molecules with a larger aspect ratio, such as butane, there is no orientationaldisorder transition and tilting of the molecules from the adsorption plane creates sufficient free area for the monolayer to melt. The promotion of molecules to a second layer is energetically less favorable for these systems.

The motion in the azimuthal angle of the N-N bonds above the disorder transition may be characterized as a hindered rotation, which evolves into a nearly free rotation at temperatures above $50 \mathrm{~K}$. This is seen both in the static distribution functions and in the frequency spectra of the mode.

We were able to treat the melting of the monolayer

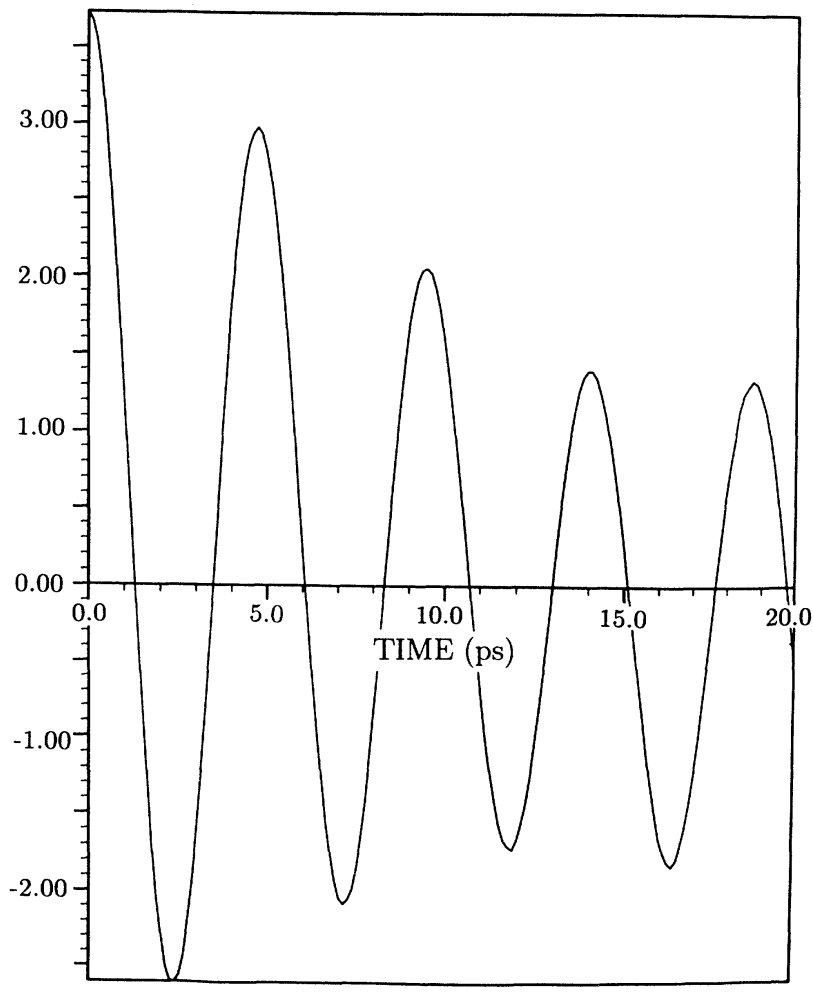

(a)

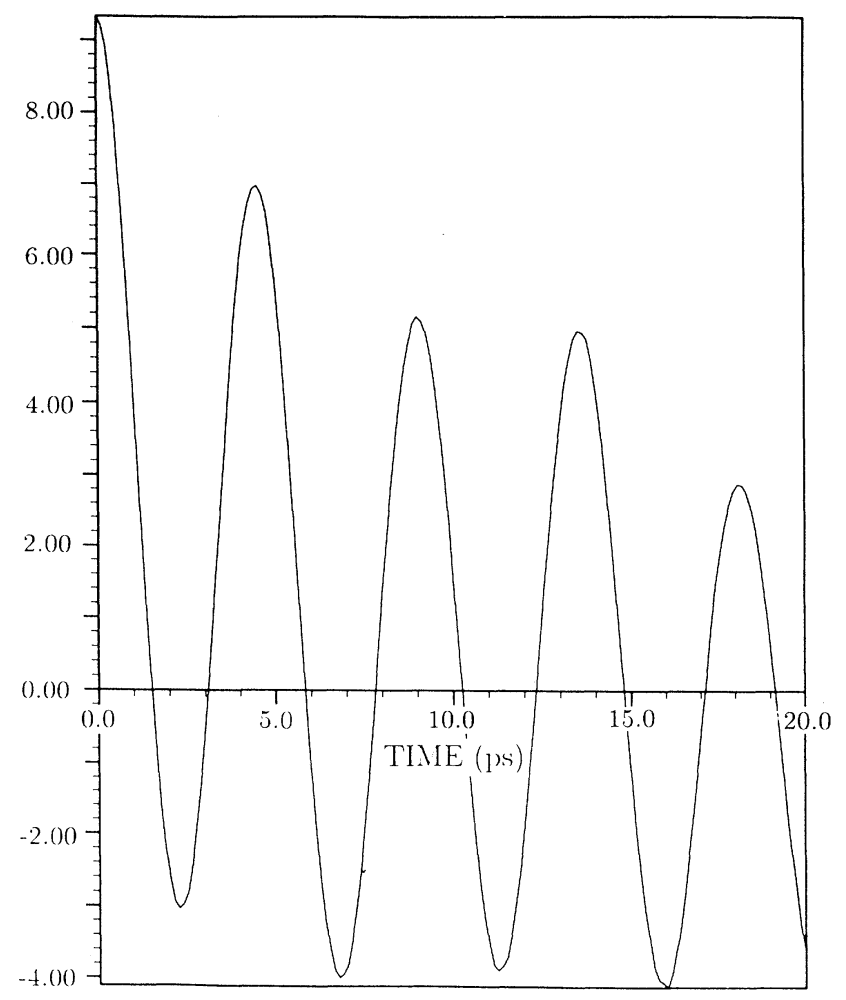

(b)

FIG. 14. One-phonon approximation to the intermediate coherent scattering function $I_{\text {coh }}^{\prime}(\kappa, t)$, for the center of mass motion at temperature $T=70 \mathrm{~K}\left(T / T_{\text {melt }}=0.959\right)$. The reciprocal lattice vectors for (a) and (b) are the same as in Fig. 13 . The period of the oscillation is distinctly longer than in that figure. 
solid in these simulations, but a few degrees above the melting temperature a significant amount of molecules desorbs. Even in the fluid the lateral motion may be characterized as a hindered translation, since the thermal average of the corrugation energy remains nonzero just above the melting point. A treatment of the high temperature fluid requires the establishment of an equilibrium between adsorbing and desorbing molecules, which leads to a reduced density of the first layer of the film. This has been done at 75 and $80 \mathrm{~K}$ and we have determined diffusion constants at the two temperatures. From the $80 \mathrm{~K}$ data, preliminary values for diffusion constants in the first layer and the second layer have been reported.

The temperatures of both the orientational disorder transition and the melting transition are lower than the experimental values and the values obtained in other simulations. Our as yet unpublished simulations of submonolayer films also lead to low melting temperatures. We have evidence that the calculated transition temperatures are brought into closer agreement with experiment if the McLachlan substrate-mediated interaction is suppressed. While it is known $^{11}$ that there are significant adsorption-induced repulsions for $\mathrm{N}_{2}$ /graphite, the specification of the adsorption-induced interactions for the nitrogen/graphite system seems to be seriously incomplete.

\section{ACKNOWLEDGMENTS}

We should like to thank $H$. Taub for continued interest in this work and for interesting and constructive

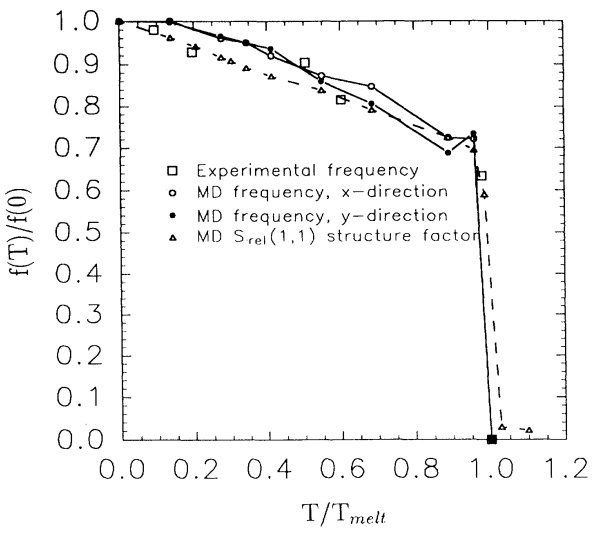

FIG. 15. Calculated gap frequencies $f(T)$ for translational motion in the $x$ and $y$ directions relative to the frequency at 0 $\mathrm{K}, f(0)$, and experimental inelastic neutron scattering results as a function of the relative temparature $T / T_{\text {melt }}$. Also shown as a dashed line is the relative structure factor $S_{\text {rel }}(1,1)$ with respect to the structure factor $S(1,1)$ at $0 \mathrm{~K}$.

discussions. L.W.B. thanks the Fysisk-Kemisk Institut for its hospitality during part of this work and acknowledges partial support by the National Science Foundation Grant No. DMR-9120199 and by the Technical University of Denmark.
${ }^{1}$ Phase Transitions in Surface Films 2, edited by H. Taub, G. Torzo, H.J. Lauter, and S.C. Fain, Jr. (Plenum, New York, 1991).

2 N.D. Shrimpton, B. Bergersen, and B. Joos, Phys. Rev. B 29, 6999 (1984); R.J. Gooding, B. Joos, and B. Bergersen, ibid. 27, 7669 (1993).

${ }^{3}$ F.F. Abraham, W.E. Rudge, D.J. Auerbach, and S.W. Koch, Phys. Rev. Lett. 52, 445 (1984); S.W. Koch and F.F. Abraham, Phys. Rev. B 33, 5884 (1986).

${ }^{4}$ H. J. Lauter, V. L. P. Frank, H. Taub, and P. Leiderer, in Proceedings of the 19th International Conference on Low Temperature Physics, edited by D.S. Betts [Physica B 165\& 166, 611 (1990)].

${ }^{5}$ F.Y. Hansen, V.L.P. Frank, H. Taub, L.W. Bruch, H.J. Lauter, and J.R. Dennison, Phys. Rev. Lett. 64, 764 (1990).

${ }^{6}$ F.Y. Hansen, L.W. Bruch, and S.E. Roosevelt, Phys. Rev. B 45, 11238 (1992).

${ }^{7}$ A. Vernov and W.A. Steele, Langmuir 8, 155 (1992).

${ }^{8}$ R. Chen, P. Trucano, and R.F. Stewart, Acta Crystallogr. Sect. A 33, 823 (1977).

${ }^{9}$ R.D. Etters, M.W. Roth, and B. Kuchta, Phys. Rev. Lett. 65, 3140 (1990); M. Roth and R.D. Etters, Phys. Rev. B 44, 6581 (1991); R.D. Etters, R. Kuchta, and J. Belak, Phys. Rev. Lett. 70, 826 (1993).

${ }^{10}$ B. Kuchta and R. D. Etters, Phys. Rev. B 36, 3400 (1987).

${ }^{11}$ M. J. Bojan and W. A. Steele, Langmuir 3, 116 (1987).

${ }^{12}$ G. Cardini and S. F. O'Shea, Surf. Sci. 154, 231 (1985).

${ }^{13}$ R. M. Lynden-Bell, J. Talbot, D. J. Tildesley, and W. A.
Steele, Mol. Phys. 54, 183 (1985).

${ }^{14}$ V. R. Bethanabotla and W. A. Steele, J. Chem. Phys. 91, 4346 (1989).

${ }^{15}$ N. D. Shrimpton and W. A. Steele, Phys. Rev. B 44, 3297 (1991).

${ }^{16}$ W. C. Gear, Numerical Initial Value Problems in Ordinary Differential Equations (Prentice Hall, Englewood Cliffs, NJ, 1971).

${ }^{17}$ D.J. Evans, W.G. Hoover, B.H. Failor, B. Moran, and A.J.C. Ladd, Phys. Rev. A 28, 1016 (1983); R. Edberg, D.J. Evans, and G.P. Morriss, J. Chem. Phys. 84, 6933 (1986); W. F. van Gunsteren and H. J. C. Berendsen, Mol. Phys. 34, 1311 (1977); J.P. Ryckaert, G. Ciccotti, and H.J.C. Berendsen, J. Comput. Phys. 23, 327 (1977).

${ }^{18}$ F.Y. Hansen, J.C. Newton, and H. Taub, J. Chem. Phys. 98, 4128 (1993).

${ }^{19}$ H.-Y. Kim and W. A. Steele, Phys. Rev. B 45, 6226 (1992).

${ }^{20}$ E. J. Meijer, D. Frenkel, R. LeSar, and A. J. C. Ladd, J. Chem. Phys. 92, 7570 (1990).

${ }^{21}$ O. G. Mouritsen and A. J. Berlinsky, Phys. Rev. Lett. 48, 181 (1982); O. Opitz, D. Marx, S. Sengupta, P. Nielaba, and K. Binder, Surf. Sci. 297, L122 (1993).

${ }^{22}$ M. P. Allen and D. J. Tildesley, Computer Simulation of Liquids (Clarendon Press, Oxford, 1987), Sec. 6.3.

${ }^{23}$ W. Marshall and S.W. Lovesey, Theory of Thermal Neutron Scattering (Oxford University Press, London, 1971).

${ }^{24}$ A. A. Maradudin and A. E. Fein, Phys. Rev. 128, 2589 (1962).

${ }^{25}$ C.S. Murthy, K. Singer, M.L. Klein, and I.R. McDonald, 
Mol. Phys. 41, 1387 (1980).

${ }^{26}$ A.D. McLachlan, Mol. Phys. 7, 381 (1964).

${ }^{27}$ L.W. Bruch, J. Chem. Phys. 79, 3448 (1983).

${ }^{28}$ W.A Steele, J. Phys. (Paris) Colloq. 38, C4-61 (1978).

${ }^{29}$ W. A. Steele, Surf. Sci. 36, 317 (1983).

${ }^{30}$ W.E. Carlos and M.W. Cole, Surf. Sci. 119, 21 (1982)

${ }^{31}$ Y.P. Joshi and D.J. Tildesley, Mol. Phys. 55, 999 (1985).

32 A.D. Buckingham, Q. Rev. 13, 183 (1959).

${ }^{33}$ D. B. Whitehouse and A. D. Buckingham, J. Chem. Soc. Faraday Trans. II 89, 1909 (1993).

${ }^{34}$ J. Larese, L. Passell, and B. Ravel, Can. J. Chem. 66, 633 (1988), and references cited therein.

${ }^{35}$ R.D. Diehl and S.C. Fain, Jr., Surf. Sci. 125, 116 (1983); A.D. Migone, H. Kim, M. H. W. Chan, J. Talbot, D.
J. Tildesley, and W.A. Steele, Phys. Rev. Lett. 51, 192 (1983).

36 T. Chung and J. Dash, Surf. Sci. 66, 559 (1977); M. Chan, A. Migone, K. Miner, and Z. Li, Phys. Rev. B 30, 2681 (1984).

37 T. Shirikami, A. Inaba, and H. Chihara, Thermochim. Acta 163, 233 (1990); A. Inaba, T. Shirikami, and H. Chihara, J. Chem. Thermodyn. 23, 461 (1991).

${ }^{38}$ D. R. Nelson and B. I. Halperin, Phys. Rev. B 19, 2457 (1979); T. M. Hakim, H. R. Glyde, and S. T. Chui, ibid. 37, 974 (1988).

39 J. P. Coulomb, M. Bienfait, and P. Thorel, J. Phys. (Paris) 42, 293 (1981). 